NBSIR 85-3105

\title{
Principles of Quality Assurance of Chemical Measurements
}

John K. Taylor

U.S. DEPARTMENT OF COMMERCE

National Bureau of Standards

Center for Analytical Chemistry

Gaithersburg, MD 20899

February 1985

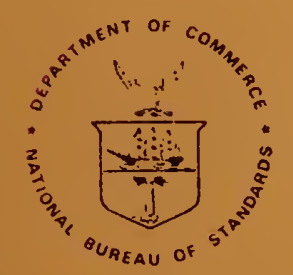

$\mathrm{QC}$

S. DEPARTMENT OF COMMERCE

100

456

$85-3105$

1935

IIONAL BUREAU OF STANDARDS 
Gaithersburg, Maryland 20899

Feburary 1985 



\section{ABSTRACT}

The general principles of quality assurance of chemical measurements are discussed. They may be classified as quality control -- what is done to control the quality of the measurement process, and quality assessment -what is done to evaluate the quality of the data output. Quality assurance practices are considered as a hierarchy with levels progressing from the analyst, the laboratory, the project, to the program. The activities of each level are different and depend upon the ones beneath it. Recommendations are presented for developing credible quality assurance practices at each level. An appendix contains outlines that may be used to develop the various documents associated with a quality assurance program. 
This document was prepared as part of a cooperative program between the National Marine Pollution Program Office and the Center for Analytical Chemistry, National Bureau of Standards. It discusses the general principles of quality assurance of measurements and contains comprehensive generic guidelines for all aspects of quality assurance efforts ranging from those of an individual laboratory to those of a monitoring program.

In preparing this document, it was recognized that specific guidelines are not only virtually impossible but may be counterproductive for quality data. Quality assurance is not like a software package that can be purchased and utilized but rather needs to be developed for each individual measurement situation and by each individual laboratory for its own use. What might be superfluous in one case could be inadequate for another purpose. Actually, the process of development of a credible quality assurance program is educational and could even be a major benefit in itself.

Most well-managed laboratories will find that what they are now doing may be most of what is necesary to be done. However, most will benefit by adopting a more systematic approach to measurement, by improvement of their documentation, and by a more rigorous statistical evaluation of their data outputs. The result will be measurement data to which statistically supported limits of uncertainty can be assigned, and this should be an overall objective of every laboratory.

It is hoped that these guidelines will be helpful to all laboratories as they strive to develop their individual quality assurance programs. It is realized that all of the suggestions contained may not be applicable to every laboratory but it is hoped that there are no serious omissions that would seriously affect data quality. Accordingly, each laboratory is urged to study the contents of this report, to accept those parts which are pertinent to its own measurement situation and which will be most useful to it in its efforts to produce sound and dependable data.

The financial support of the National Marine Pollution Program and the encouragement of its Director, Dr. Andrew Robertson, during the preparation of this document are gratefully acknowledged. 
1. Introduction. . . . . . . . . . . . . . . . . . 1

2. Measurement and Calibration .............. 3

2.1 Selection of Methodology ............. . . 3

3. The Quality Assurance Concept . . . . . . . . . . . 5

4. Statistical Control . . . . . . . . . . 8

5. Quality Control Techniques ............ 9

5.1 Competence . . . . . . . . . . . . . . . 9

5.2 Suitable Facilities and Equipment. . . . . . . . . . 9

5.3 Good Laboratory Practices and Good Measurement Practices . 10

5.4 Standard Operations Procedures . . . . . . . . . 10

5.5 Protocols for Specific Purposes. . . . . . . . . 12

5.6 Other Quality Control Techniques .......... 12

5.6.1 Inspection ............. 12

5.6 .2 Documentation ............. 13

5.6 .3 Training ............. 14

6. Quality Assessment Techniques . . . . . . . . . . 15

7. Control Charts. . . . . . . . . . . . . 18

8. Samples and Sampling . . . . . . . . . . . . 20

8.1 Uncertainty of the Sample . . . . . . . . . . 20

8.2 Quality Control of Sampling . . . . . . . . . . 20

8.3 Quality Assessment of Sampling . . . . . . . . . 21

8.4 Chain-of-Custody ............... . . 21

9. Levels of Quality Assurance . . . . . . . . . . . . 22

10. Quality Assurance Policy . . . . . . . . . . . 23

11. Planning for Quality Assurance . . . . . . . . . . . 24

12. Development of a Quality Assurance Program. . . . . . . . . 26

13. Quality Assurance Manual. . . . . . . . . . . . 28

14. Quality Assurance of a Measurement Program. . . . . . . . . 29

15. Protocols for Specific Purposes . . . . . . . . . . 31

15.1 Designation of Principal Investigator ........ . 31

15.2 Definition of Problem . . . . . . . . . . . 31

15.3 Specification of Model ............. . . . 31

15.4 Specification of Samples ............ 31

15.5 Specification of Data Base . . . . . . . . . . 32

15.6 Specification of Methodology ............ . 32

15.7 Specification of Controls . . . . . . . . . . 32

15.8 Release of Data ............... . . . 32

16. Quality Assurance of a Monitoring Program . . . . . . . . . 34

17. Functions of a Reference Laboratory . . . . . . . . . . . 37

18. Bibliography of Selected Quality Assurance Documents..... . 35 
Appendix A. Program Documentation . . . . . . . . . . 47

A.1 Example-Quality Assurance Program Document . . . . . . . 47

A:2 Outline-Development of a Quality Assurance Protocol for a Specific Purpose (PSP) ............. 55

A.3 Outline-Content of a Quality Assurance Protocol for a Specific Purpose ............. . . 56

A.4 Outline-Organizing and Implementing a Monitoring Program . 59

A.5 Outline-Functions of a Reference Laboratory . . . . . . 60

Appendix B. Data Quality Objectives . . . . . . . . . 62

Appendix C. Terminology Used in Quality Assurance . . . . . . 64 


\section{LIST OF FIGURES}

Pages

Figure 1. Production Process Quality Assurance . . . . . . . . 6

Figure 2. Measurement Process Quality Assurance . . . . . . . 6

Figure 3. Methodology Selection . . . . . . . . . . . 11

Figure 4. Quality Control by Inspection . . . . . . . . . 12

Figure 5. Quality Assurance Perspective . . . . . . . . . 25

Figure 6. Quality Assurance of a Measurement Program . . . . . . 29

Figure 7. Analytical Problem Solving . . . . . . . . . . 30

Figure 8. Cooperative Measurement Program . . . . . . . . . 39

Figure 9. Quality Assurance Function of a Reference Laboratory . . 41 
$\underline{\text { Pages }}$

Table 1. Basic Elements of Quality Control .......... 9

Table 2. Quality Assessment Techniques . . . . . . . . 15

Table 3. Protocols for Specific Purposes . . . . . . . . . 33

Table 4. Functions of a Reference Laboratory in National

Measurement/Monitoring Programs . . . . . . . 38 


\section{INTRODUCTION}

The demand for measurement data is ever increasing. Decisions need to be made on such questions as the suitability of a material for an intended purpose, the quality of the environment, and the health of individuals. The vast majority of such measurements entail chemical analyses in which some chemical property of the material or object of interest is measured.

Dramatic changes have taken place in chemical analysis during the past several decades. Most measurements are now being made using complex instruments in sophisticated processes. Many of the operations previously done manually are now automated. Moreover, measurements made by several analysts and/or laboratories often need to be interrelated for use in a decision process. Monitoring programs of regional, national, or international scope may be involved. All of this places stringent requirements for the reliability and compatibility of the data.

The quality of data is ordinarily evaluated on the basis of their uncertainty when compared with end-use requirements. If data have consistency and their uncertainty is small when compared to the requirements, they are considered to be of adequate quality. When excessively variable or the level of uncertainty exceeds the needs, the data may be said to be of low or inadequate quality. The evaluation of data quality is thus a relative determination. What is high quality for one situation could be unacceptable in another.

The quality of chemical data may be judged on the basis of two aspects: the accuracy of identification of the parameter measured; and, the numerical accuracy. The qualitative identification must be without reasonable doubt. The analyst must state precisely what was measured and be able to prove it. While empirical measurements, defined by a specific apparatus or methodology may be useful in some situations, they must be specified as such and used with due consideration of their limitations.

Quantitative measurements are always estimates of the value of the measurand and involve some level of uncertainty. The measurements must be made so that this uncertainty can be assigned within a stated probability. Without such an assignment, no use can be made of the data in any logical sense. To achieve this, measurements must be made in such a way to provide statistical predictability. The experience of metrologists has demonstrated that such is achieved best by a well-designed and consistently implemented quality assurance program.

The above discussion concerns the quality of measurement data, and indeed the quality of individual measured values. When considered collectively as a body of numerical information for regulatory use, for discussion, or inference, additional quality descriptors may be useful. Thus the Environmental Protection Agency proposes [45]:

Completeness - A measure of the amount of data obtained from a measurement process compared to the amount that was expected to be obtained under the conditions of measurement. 
Representativeness - The degree to which the data accurately and precisely represent a characteristic of a population parameter, variation of a property, a process characteristic, or an operational condition.

Comparability - Expresses the confidence with which one data set can be compared to another.

While difficult to quantify, such characteristics need to be considered when evaluating the usefulness of a set of data for scientific interpretation.

The production of data of adequate quality is a common commitment of most laboratories. However, it is pursued differently:

- in various organizations;

- within laboratories in the same organization;

- within groups in the same laboratory;

- from time-to-time by the same individual.

It is understandable why this is so. The kind and amount of quality assurance effort is related to the kind of work that is done, the knowledge, skill and expertise of analysts, and the quality requirements for the data. In many measurement situations, it is assumed that measurement uncertainty is insignificant compared to the end use of the data. In such cases, the measurement process may not be optimized, and the uncertainty of the data is not fully evaluated. The adoption and implementation of a uniform credible quality assurance policy within organizations and across organizational units, should minimize much of the data uncertainties that exist and harmonize data outputs.

It should be recognized that quality assurance is not a system of software that can be installed but must be developed to satisfy individual needs. W. Edwards Deming, one of the acknowledged leaders of the quality assurance effort of industry, has said, "An obstacle that ensures disappointment is the supposition, all too prevalent, that quality control is something you install.... Actually, to be successful in any company, it must be a learning process.... with accumulation of knowledge and experience under competent tutelage" (14).

With due appreciation of the above, one can profit from the experience of others, while developing a quality assurance program for a specific need. The information that follows summarizes the experience and thinking of the measurement community on the requirements for reliable measurements. It is presented with the premise that it will provide guidance to laboratories in the development of unique, individualized quality assurance programs that at the same time, will have interlaboratory consistency. 
Measurement is essentially a comparison process. Units are defined by convention or consensus agreement and are established by suitable standards. Comparators in the form of graduated scales or measuring devices incorporating sensors are used to compare an unknown for which some property needs to be measured with a standard for which that property is believed to be known with sufficient reliability.

While all measurement involves some kind of comparison, the measurement may be described as absolute when only basic standards are involved and/or are interrelated by fundamental relationships. When derived standards are involved and/or related by empirical or inferrential relationships, the measurement may be described as comparative. The majority of modern analytical measurements are comparative, although absolute measurements such as gravimetry and coulometry are actively used.

In every measurement situation, several assumptions are explicitly or implicitly involved. The first is that the property of interest is measurable by the measurement process. A second assumption is that suitable standards are available. A third assumption is that the measurement or intercomparison is meaningful and can be reproduced within describable limits. The reliability of the measurement process will depend upon the validity of these assumptions. Judgement of such will require a comprehensive knowledge of the methodology, of the matrix measured, and demonstration of the stability and performance characteristics of the measurement process.

The influence of the matrix on the measurement process always needs clarification. The analytical chemist must demonstrate that this is negligible or is adequately compensated, else the measured values can hardly have significance. Calibration procedures including the use of matrix-matching standards, spiking, and standard-additions may be employed but their adequacy must be demonstrated. Matrix modification can also be used in some cases. Removal of the analyte from the matrix and transferring it to a more benign one can provide increased sensitivity and minimize matrix effects. In each case, the analytical uncertainties introduced must be understood and taken into account in reporting results.

Too often, calibration is done in a perfunctory manner. Analysts are urged to give greater attention to the calibration process and to carefully consider the bias and random error that it may introduce. Bias can result if the calibrants used are inappropriate (matrix-mismatch) or if their compositions are inaccurately defined. Drif'c of a measurement system between calibrations is a source of bias, hence calibration frequency must be carefully considered. Calibrations are often considered as "errorless" and rarely repeated except at intervals. As a result, each individual calibration may be based on a very few (sometimes zero) degrees of freedom. When calibration uncertainty becomes significant, as in ultra-trace analysis, it may become a major component of variance of the measurement system.

\subsection{SELECTION OF METHODOLOGY}

The methodology used must be appropriate and adequate for the measurements that are required. In order to decide the appropriateness of the methodollogy, advance information is needed on the parameters to be measured, the 
level(s) of concentration expected, and the minimum differences among samples that should be distinguishable. The operational characteristics of the proposed methodology must be known and should equal or exceed the requirements for the data. In addition, the repeatibility and reproducibility should be sufficient to minimize the number of replicate measurement that should be made. The selectivity should be adequate or ways to eliminate interference from other constituents of the sample must be available.

The limit of detection is an important consideration since it sets a lower limit for qualitative use of the methodology. The limit of quantitation defines the lower limit where quantitative measurements can be made with reasonable $( \pm 30 \%)$ confidence (41). The limit of detection must be suitable in most trace situations and the limit of quantitation should be less than the levels at which quantitative data are required.

In addition to the above, it is desirable that the methodology is easy to use, has a low unit-cost of operation, provides a rapid rate of analysis, is useful over a wide range of concentrations, and is relatively insensitive (rugged) to reasonable variations of its operational parameters.

A first requirement for a valid method (49) is that its operational characteristics are adequate for the data requirements. A second requirement is that a standard operations procedure (SOP) has been developed so that it can be used in a specified manner by a competent analyst. Both of these factors, and especially the latter, may be judged by collaboratively testing the methodology.

It is obvious that valid data cannot be expected unless valid methodology is used, but this does not guarantee reliable data. The laboratory/analyst must demonstrate competence and must use an appropriate quality assurance program to support the quality of the data. 


\section{THE QUALITY ASSURANCE CONCEPT}

Every experimenter knows that the absolute accuracy of any measurement cannot be guaranteed. Yet data are for use, so they must be trustworthy. Their quality must be defined, which means that the limits of confidence must be evaluated and stated. The objective of a quality assurance program is to provide a statistical basis for the assignment of such limits.

Quality assurance consists of two separate but related activities, quality control and quality assessment. Both must be operational and coordinated. The following definitions are offered.

Quality assurance: A system of activities whose purpose is to provide to the producer or user of a product or a service the assurance that it meets defined standards of quality with a stated level of confidence.

Quality control: The overall system of activities whose purpose is to control the quality of a product or service so that it meets the needs of users. The aim is to provide quality that is satisfactory, adequate, dependable, and economic.

Quality assessment: The overall system of activities whose purpose is to provide assurance that the overall quality control job is being done effectively. It involves a continuing evaluation of performance of the production system and the products produced.

The objective of quality control is to fine tune and maintain a measurement process in a desired state of stability and reproducibility. In such, the process can be considered to have a high degree of similarity to an industrial production process, with the ability to produce objects (in this case data) with a high degree of reproducibility. Once this is established, the precision may be defined and any biases can usually be identified and eliminated or compensated, so that requisite data quality is achieved. Quality assessment provides the evidence that this has been accomplished.

The general approach to quality assurance of the measurement process parallels that for the industrial production process and borrows concepts and techniques proven trustworthy for the latter, to the extent possible. In industry, the procedure may be called statistical product control. In metrology, the procedure may be called statistical measurement control.

The quality assurance procedure followed in a typical production process is illustrated in figure 1. Experience dictates the kind and degree of control that must be achieved and maintained to obtain products of desired quality. Random samples of the output are tested or inspected with respect to spec1fications, in order to release or accept the production lot, to reject $1 t$, or to take corrective actions in the process.

The quality assurance aspects of a typical measurement process are illustrated in figure 2. Quality control procedures are used to tune and maintain the system in a state of statistical control in which it may be considered as capable of generating an infinite number of measurements of any material, of which the data of the moment are a typical sample. Quallty assessment procedures are then used to evaluate the quality of the data that are produced. Unfortunately, it is generally impossible, intrinsically, to 
evaluate fully the quality of the data on unknown test materials. However, if the measurement system is in a state of statistical control, known test samples such as reference materials (RM) may be measured concurrently and the results compared with the reference values. Such a comparison can be used to evalutate the performance of the measurement system and permit inferences to be made on the quality of the data for test samples.

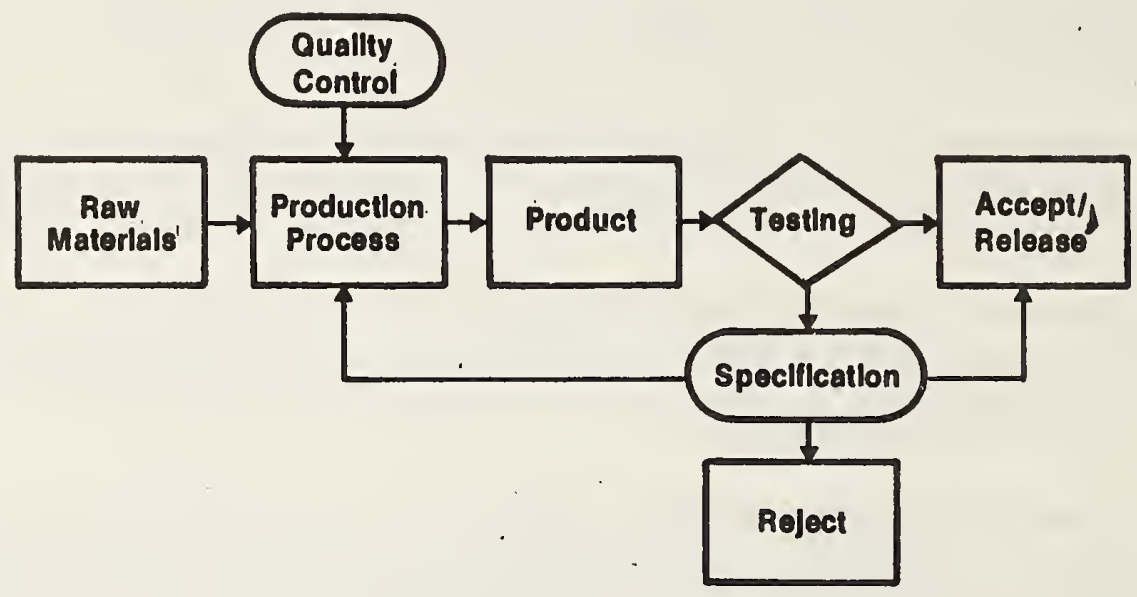

Figure 1. Production Process Quality Assurance

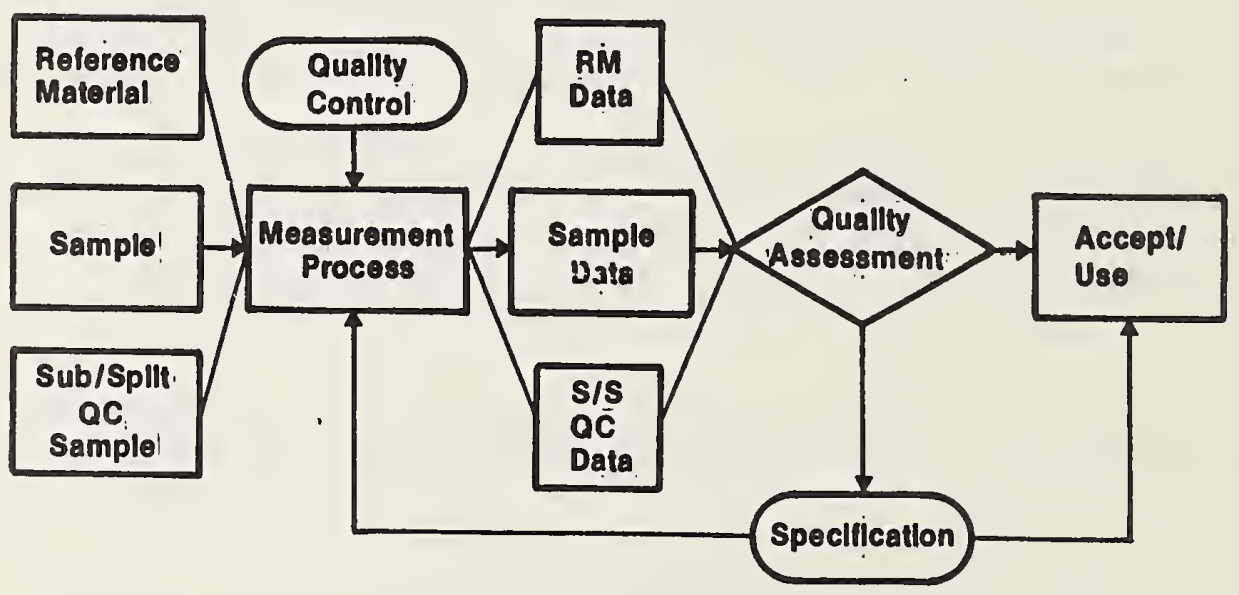

Figure 2. Measurement Process Quality Assurance

Quality assurance activities may be considered as a hierarchy progressing in an ascending order [51] from the analyst $\rightarrow$ laboratory $\rightarrow$ project $\rightarrow$ program. Each level requires different activities and has different responsibilities which will depend on those below it. The analyst has the closest control of the measurement process and the responsibilites are largely technical in 
nature. The laboratory provides facilities and human resources and has supervisory and managerial responsibilities. The success of a project will depend on all of these and in addition will hinge on the soundness of the technical concepts and planning to execute a valid experimental approach. The project leader, of course, will have major responsibilities in this regard. A program may be considered as a collection of projects which must be properly conceived, coordinated, and managed for which the program manager has prime responsibility. Intercalibration of the data-producing activities is a major requirement for such a program. It is obvious that the quality assurance procedures will be different for each level of activity and they should be specifically designed. Each level may be considered as a subcontractor for the ones above it. If its quality assurance practices are appropriate and well executed, a minimal amount of inspection or checking should be required by the upper levels, which then can devote their efforts largely to the quality assurance activities peculiar to their specific responsibilities. Each level must inspire confidence in the levels above it and provide supporting evidence for the quality of its outputs. The reader is referred to reference 51 where the concepts mentioned above are discussed in greater detail. 


\section{STATISTICAL CONTROL}

Everyone requiring or producing measured values would like them to be accurate. Yet, it is generally recognized that measurement is a process of estimation and that some degree of uncertainty is involved. It is axiomatic that a meaningful measurement is reproducible yet it is common experience that repetitive measurements will disagree and that the means of such, while agreeing better, will also disagree. In fact, too close agreement of ten indicates a rounding-off either by the measurement process or in the reporting of the data.

It is becoming increasingly recognized that limits of uncertainty (error bars) must be established and associated with every data point so that the strength and weakness of every decision based on them may be evident. This supports valid interpretation and prevents over-interpretation of data. The experimenter or user of data may need to answer such questions as:

- are differences in measured values significant?

- how close would an additional value be expected to agree with the data already obtained?

- what limits of confidence can be assigned to a measured value?

- are the performances of two laboratories/analysts significantly different?

- are the results obtained using one method/instrument significantly different from those obtained by another?

- do estimates of precision obtained on several occasions differ?

These and similar questions cannot be answered categorically but only on a probability basis, which requires that the related measurements must be in a state of statistical control.

Statistical control means that the output of a measurement process is predictable by statistical concepts. The measurement errors have attained a state of randomness so that predictive statistical models validly represent the data. (Biases may or may not be present, but their identification will be facilitated, as well).

The prerequisite for statistical control is a stable measurement process. In such, the assignable causes of variation are kept in control by the quality control program and the measurement operations are optimized and implemented by standardized procedures so that chance causes of random error are minimized and/or acceptable. The output of such a system will be expected to have a limiting mean and a definite distribution from which statistical predictions can be made.

Eisenhart has stated, "To qualify as a measurement process, a measurement operation must have attained what is known in industrial quality control language as a state of statistical control. Until a measurement operation has been 'debugged' to the extent that it has attained a state of statistical control, it cannot be regarded in any logical sense as measuring anything at all" (15). 


\title{
5. QUALITY CONTROL TECHNIQUES
}

Quality control techniques include all practices and procedures that lead to statistical control of the measurement process. The basic elements of quality control are listed in table 1. The list proceeds from the general to the specific and each element depends on the ones above it [52].

\author{
Table 1. Basic Elements of Quality Control \\ Technical Competence of Staff \\ Suitable Facilities and Equipment \\ Good Laboratory Practices (GLPS) \\ Good Measurement Practices (GMPs) \\ Standard Operations Procedures (SOPS) \\ Protocols for Specific Purposes (PSPS) \\ Inspection \\ Documentation \\ Training
}

\subsection{COMPETENCE}

The competence and expertise of the technical staff is the first requirement for quality measurements. Despite the increasing sophistication of modern instrumentation, technical judgement, experience, skill, and even the professional attitudes of the staff are very important for reducing and maintaining measurement variability at acceptable levels. The laboratory staff must have at least a minimum level of competence commensurate with the skill requirements of the analytical discipline. Only in the most repetitive of measurement situations can the chemical measurement process be reduced to a mechanical routine. It is common knowledge that measurement competence increases as experience is gained with most methods, and that laboratories, seemingly equal with respect to facilities and equipment, can differ significantly in the quality of their data output.

\subsection{SUITABLE FACILITIES AND EQUIPMENT}

Modern chemical analysis typically requires specialized facilities, apparatus, and equipment, and success or failure often can be traced to their adequacy and mode of utilization. Thus ultra-trace analysis hardly can be done without ultra-clean laboratories, and toxic substance analysis may require the use of containment areas. Humidity and temperature control are recognized prerequisites for reliable measurements in some areas of analysis and modern data processing and management systems are indispensible for some and are fast becoming so for most other analytical techniques.

Specialized apparatus and equipment are rapidly displacing general-purpose equipment so that certain kinds of analytical measurements are limited to laboratories where such are available. In some cases, apparatus dedicated to a specific analytical problem may be needed to minimize contamination and memory effects. 
Not only are such facilities and equipment required, but they must be adequately maintained. Prescribed maintenance schedules, calibrations, and good housekeeping practices must be followed to ensure the quality of the work output.

With such dependency on facilities and equipment, their use can engender unmerited reliance and even false security by both the producer and user of the data. It is a fair statement that suitable facilities and equipment are necessary but not sufficient for the production of quality data. They must be backed up by the other components of a quality assurance system, specifically designed for the analytical problems of concern. The complex nature of the problems that can be solved by such facilities and equipment increases, rather than decreases, the need for quality assurance of the measurement process.

\subsection{GOOD LABORATORY PRACTICES AND GOOD MEASUREMENT PRACTICES}

Good laboratory practices (GLPs) and good measurement practices (GMPs) embrace the total experience of analysts in the making of good measurements. GLPs refer to the general practices that relate to many if not all of the measurements made in a laboratory. They are technique-independent and address such subjects as maintenance of facilities, records, sample handling, reagent control, and cleaning of laboratory glassware. Good measurement practices (GMPs) are technique specific. They can be extensions of GLPs to the requirements of specific measurement techniques or they may be completely independent of such.

The subjects for GLPs and GMPs are best identified and the contents developed by the laboratory personnel on the basis of their experience and that of their peers. GLPs and GMPs must be documented and formally implemented in a laboratory if good measurements are to result. Too often, GLPs and GMPs are assumed to be common knowledge and unnecessary subjects for documentation. It is true that many of the things done in a laboratory do not need to be formalized. Indeed, it would be virtually impossible to document every procedure and every step of an operation. However, critical operations can be identified and should be optimized. Any operation or sub-operation that is discovered to be an assignable cause of significant variance or bias is a prime subject for optimization and documentation.

\subsection{STANDARD OPERATIONS PROCEDURES}

Standard operations procedures (SOPS) describe the way specific operations and methods are performed. These include sampling operations, sample preparation, calibrations, measurement procedures, and any operation that is done on a repetitive basis. "Standard" means that it specifies the way the operation is to be done on each occasion, which may or may not be the procedure developed by a standards-writing organization. However, when such are avallable, laboratories are well advised to consider them since they represent peer judgement and provide a basis for comparability of data among user laboratories.

When methods are repeatedly used, there is incentive to write them up in a formal manner, but those used on an infrequent basis are often based on an analyst's "know-how". Such methodology is seldom optimized and frequently is used differently on each occasion of use. 
The practice of using unwritten methods should be discouraged. Documentation of what was done is almost always needed. When the writing is done afterthe-fact, it is likely to be brief and sketchy and possibly inaccurate. Describing a method before its use is part of planning and should be done with as much care as that used for all other aspects of a chemical analysis. colleague review of all methodology is a minimum requirement to insure understandability and hence continuity of a measurement process, as well as to receive the benefits of technical criticism. The writing need not be difficult and time-consuming if a standard format is used. This also provides a mechanism for screening for possible omissions in the descriptions and for intercomparisons in some cases.

While the use of SOPs may provide a continuity of measurement experience, no methodology should be used blindly. Its appropriateness should be considered at each time of use. If used infrequently, it may be necessary for the analyst to make sufficient preliminary measurements to validate competence and to demonstrate attainment of statistical control of the measurement process (49) on each occasion.

A recommended pattern for increasing the inventory of useful methodology of a laboratory is illustrated in figure 3. Certain of its methods will be

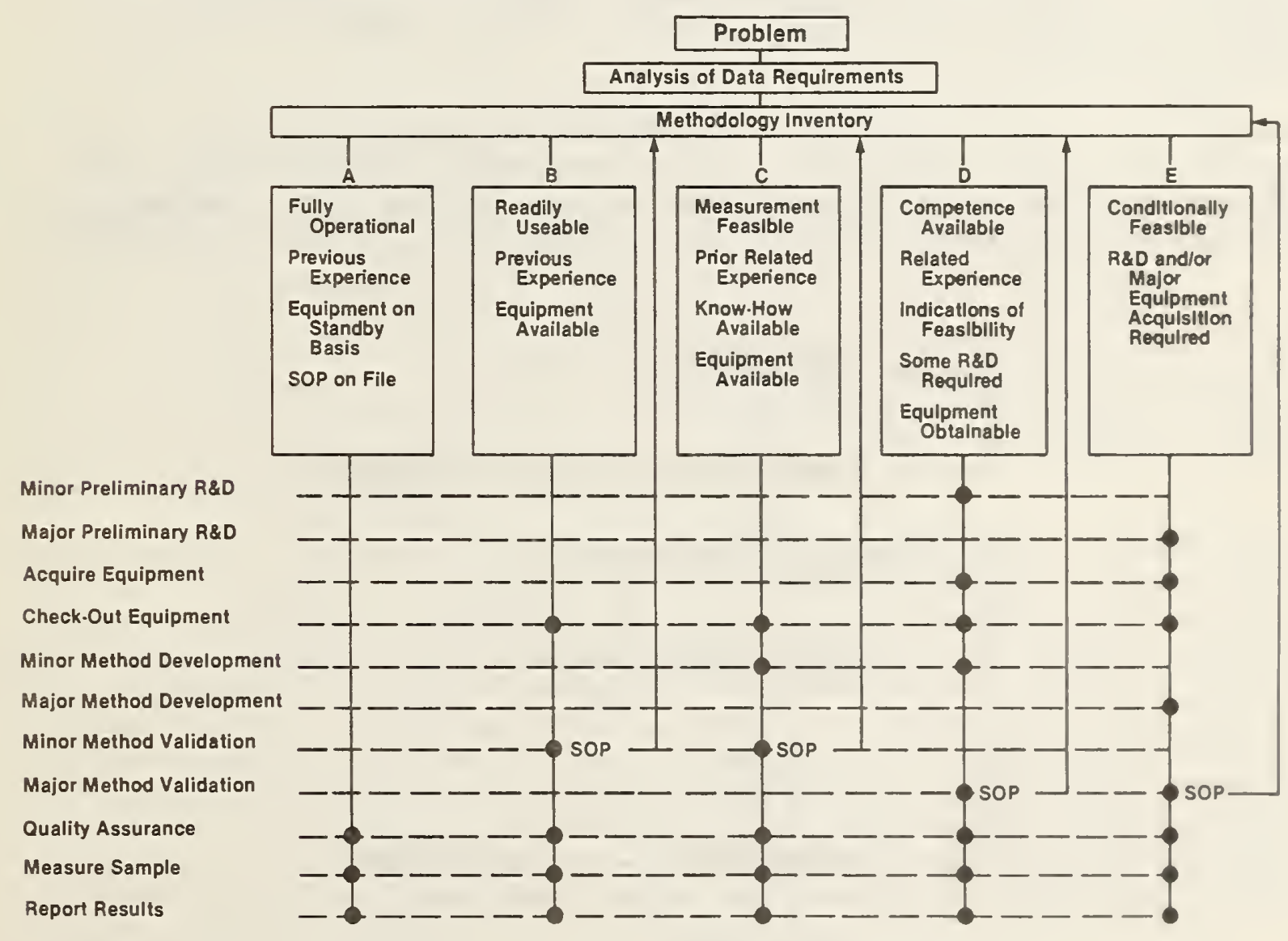

Figure 3. Methodology Selection 
in category $A$ and $B$. When new measurement challenges arise, methodology in categories $C, D$, or $E$ may be useful, but some developmental work may be required before $1 \mathrm{t}$ can be used in a trustworthy manner. If an SOP is prepared concurrent with the use of such, the methodology can move to an A or $B$ classification for future use.

\subsection{PROTOCOLS FOR SPECIFIC PURPOSES (PSPS)}

PSPS precisely define the way a specific measurement sequence, project, or program is to be conducted. The premise in the development of a PSP is that cost-effective and reliable data require a well-considered experimental plan. While quality control is the primary objective, the PSP will prescribe the quality assessment procedures to be used as well. Because of their dual nature, the concept, content, and procedure for development of PSP's will be discussed elsewhere (see Section 15) in this document.

\subsection{OTHER QUALITY CONTROL TECHNIQUES}

\subsubsection{Inspection}

Inspection is widely used in industrial operations as a quality assurance procedure. Because of its non-quantitative nature, it is most useful for quality control in the measurement process, and less useful for quality assessment purposes.

Inspection is largely a subjective examination for malfunction or for the detection of abnormal conditions. A good measurement process usually will have several places where inspection may be used beneficially as suggested by Figure 4. Samples may be inspected for missing or conflicting information

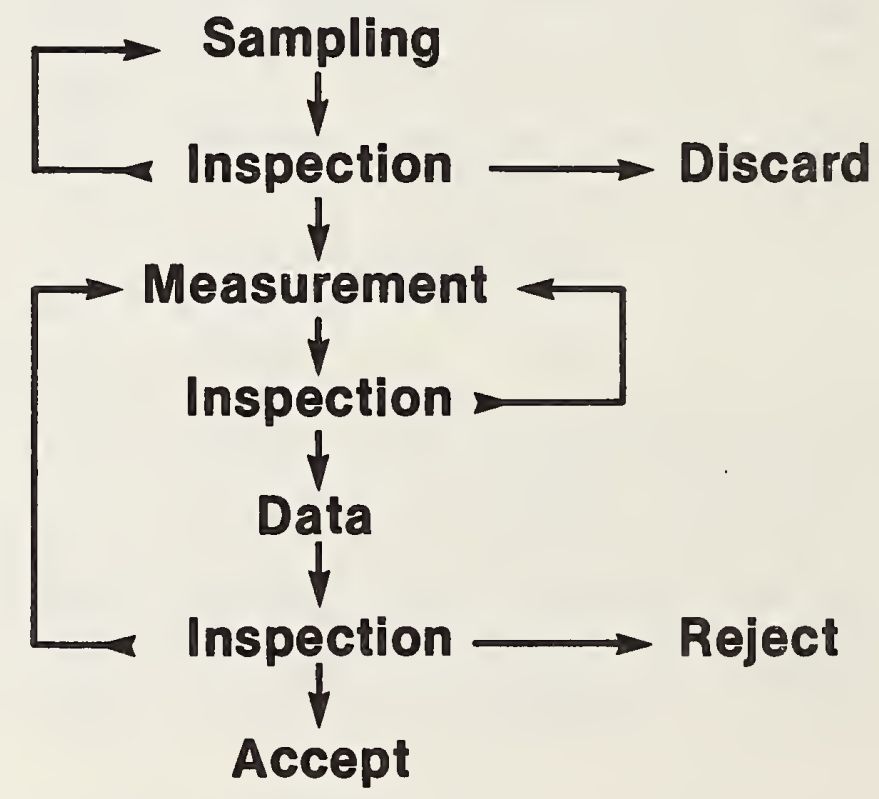

Figure 4. Quality Control by Inspection 
about the sampling process which could be cause for rejection. Also, the samples may need to meet requirements for physical appearance or phase homogeneity, for example.

The measurement process can have performance specifications for the instrumentation (stability, for example), the laboratory environment, and validity of calibrations before acceptable data are possible. Data checks may be made for reasonableness and expression in proper units, for example.

Inspections do not control quality but may prevent the reporting of faulty or questionable data. A good inspection program may make it possible to obtain additional samples or remeasurements when required and thus reduce gaps in data that might otherwise occur.

\subsubsection{Documentation}

Documentation is an important aspect of every measurement program. Data must be both technically sound and defensible. While technical soundness is a prerequisite, inadequate documentation can cast suspicion on the technical merits.

Documentation must address all aspects of the measurement process: the model, the plan, the methodology, the calibration, the sample, and the data reduction. All of these need to be tied together in a comprehensive report that shows the interrelation of each phase and summarizes the data, including all of its limitations. Conclusions, when presented, need to be fully supported by documentation.

Details of the documentation process are best addressed in the GLPS. Special instructions for documentation of the data output of measurement techniques should be included in the GMPs. The documentation requirements for a specific project or monitoring program should be planned as carefully as all other aspects of the measurement program and specified in the PSPS. In doing so, the documentation practices of all participating laboratories should be examined not only for adequacy but for compatibility. The PSPs may need to specify the format in which the data are to be presented. This may include the developement of special reporting forms.

Documentation and reporting of data must be done accurately. Transcription errors are a common problem that can be virtually eliminated by care and attention to detail. Systematic inspection and periodic review of notebooks and similar primary records are recommended to ensure the general quality of their contents. Changes or revisions of notebook entries must be justified and documented. Any changes should be made by crossing out the original entry and substituting the new value. The person making the change should initial the entry and state why the change was made. No erasures of records or data are permissible. A system for review of technical reports including countersigning by higher authority is recommended. Also, the staff members responsible for the measurements should sign reports to attest the validity of the technical contents. 


\subsubsection{Training}

Adequate education and training are prerequisites for reliable measurement capability. The latter can be distinguished from the former by the degree of specificity. The complexity of modern chemical analysis has increased so much that undergraduate education is no longer sufficient for analytical chemists. Even highly educated analytical chemists find the need from timeto-time for additional training due to advances in technology and to the adoption of new technology. On-the-job training in the quality assurance aspects of a laboratory's program is a necessity, as well. New employees should be indoctrinated in the specific quality assurance practices they must follow and refresher courses may be needed by the continuing staff. A laboratory's quality assurance manual can provide a good basis for quality assurance training.

All training should be direct; no serial training should be permitted. 
Quality assessment techniques consist of ways by which the measurement process may be monitored in order to infer the quality of the data ouput. Table 2 lists a number of approaches that may be used. The central theme is the concept of redundancy. Precision can be evaluated only by replication using stable samples of known and even unknown composition. Evaluation of bias requires the repetitive measurement of samples of known properties although fewer measurements may be required when statistical control of the measurement process is demonstrated by other means. The quality assessment techniques listed in Table 2 are classified as internal or external according to the source of the assistance needed to implement the assessment technique.

Repetitive measurement of the samples actually tested is the classical way to evaluate precision. However, this is a time consuming process if not carefully planned and it is the objective of other quality assessment techniques to minimize the number of such measurements. Duplicate measurement of an appropriate number of test samples often can provide much of the evaluation of precision that is needed and eliminate all questions of the appropriateness of the quality assurance samples.

\section{Table 2. Quality Assessment Techniques}

Internal

Repetitive Measurements

Internal Test Samples

Control Charts

Interchange of Operators

Interchange of Equipment

Independent Measurements

Definitive Method Measurements

Audits

External

Collaborative Tests

Exchange of Samples

External Reference Materials (ERM's)

Standard Reference Materials (SRM's)

Audits

Internal test samples may consist of internal reference materials (IRM's), split samples, spiked samples, and surrogates which are measured in suitable test routines to evaluate the precision of the measurement process. Measurements of all such samples and indeed of all quality assurance samples are best interpreted by the use of control charts. In this way, a few measurements of the moment can join the experience of the past to demonstrate statistical control and bolster confidence in the measurement process.

Measurement bias can be operator, equipment, and/or methodology dependent. Internal approaches that may be used to investigate such bias include interchange of operators and apparatus/equipment, measurement of selected samples by independent techniques, and comparisons of measurement results with those 
obtained, using a definitive method. The latter approach is useful when suitable methodology is available and when external aids, such as reference materials, are not available.

External evidence for the quality of the measurement process is important since it avoids the dangers of introspection. Several procedures may be followed to provide external evidence of the quality of a measurement process. These can confirm the internal evaluation of precision and provide independent assessment of any bias (or lack thereof). Activities include participation in collaborative test exercises, exchange of samples with other laboratories, and the analysis of reference materials obtained from external sources (ERMs). The National Bureau of Standards Standard Reference Materials (SRMs) $[43,44]$ are unexcelled as test materials to evaluate the measurement process when they are properly used $[12,50]$. They have the distinct advantage of wide acceptance, hence provide a basis for intercomparisons of measurement systems and test data taken under diverse conditions and by various laboratories.

From the above discussion, it should be clear that every laboratory has within-house capability to estimate the precision of its measurements. The evaluation of bias is difficult, internally, but facilitated by the use of externally available techniques. No matter what the source of information, any evidences of mal-performance, whether it be lack of precision or intolerable bias, should be carefully investigated and appropriate corrective actions should be taken as necessary. After such actions, follow-up measurements should be undertaken to verify that the problems have been eliminated.

Assessment of the accuracy of qualitative identification is difficult. The concept of control charts is not readily applicable and certified reference materials for qualitative identification are not available at detection levels where the problem of identification is most acute. Ordinarily, reliance is based on the known selectivity of the methodology and knowledge of the absence of potential interferents. The knowledge and experience of the analyst can be a key factor in decisions on detection.

Identification using independent techniques and/or several modifications of the same technique (e.g., different columns) is useful for verifying the qualitative identification of a measured analyte. Spot checks by a reference laboratory can be useful both for verification of measured analytes and to ensure confidence in question of "detect" and "non-detect".

Audits are listed as both internal and external quality assessment techniques. Two kinds of audits may be used. Systems audits consist of both systematic and spot checks of equipment, facilities, and procedures for compliance with the quality assurance plan. Performance audits depend on the use of test samples to evaluate laboratory proficiency. Monitoring networks and accrediting agencies often include audits as part of their procedures to evaluate laboratories. Laboratory management is well advised to develop its own auditing program and to conduct internal audits on both an announced and unannounced basis, whether or not required by someone else.

The use of internal test samples, particularly with a control chart, is a type of on-going performance audit. Operators should be encouraged to maintain their own control charts with real-time response capability. Supervisors and higher management levels may submit samples and keep their 
own control charts. Monitoring programs should distribute control samples and maintain control charts on a real-time basis to assure intercalibration of stations in a network. The effectiveness of the quality assurance process at any level will minimize the need and amount of effort required at higher levels to monitor the measurement process.

Systems audits should be conducted in a similar manner. Bench-level audits provide the potential for consistent implementation of the quality assurance program and again should minimize the need for higher level inspection. While the former can prevent faulty operations, the latter can usually only detect them. The approach for conducting a quality audit, described by Gaft and Richards (18), is recommended for guidance in developing such a program. 


\section{CONTROL CHARTS}

The development and use of appropriate control charts (13) is one of the most important techniques for monitoring a measurement process. The most common type involves the scheduled repetitive measurement of a typical stable test sample and plotting the results. Control limits are established within which the results are expected to lie due to random fluctuations of the measurement process. As long as they do so, the system is said to be in a state of statistical control. When the results are plotted on a sequence or time (preferable) basis, trends may be identified before "out-of-control" occurs. Similarly, the standard deviation or range of a series of measurements may be plotted as a control chart and kept in parallel with the above chart to indicate when decrease in precision occurs and hence assist in the identification of measurement problems.

It is recommended that laboratories maintain charts for the mean $(\bar{X})$ and difference $(R)$ of duplicate determinations. Furthermore, it is recommended that both warning (2s) and control (3s) limits be utilized. Such charts should be developed for samples typical of each class of tests performed by the laboratory, as a minimum. As long as the control samples are stable, knowledge of their actual composition is of lesser interest. While samples of unknown composition cannot monitor bias, they can monitor stability of the measurement process, and bias can be evaluated otherwise. Accordingly, some of the control samples used should be reference materials to facilitate the evaluation of bias.

Laboratories are urged to consider also the use of $\mathrm{R}$ (duplicate) charts utilizing the actual samples tested. While any control sample can be questioned as to its similarity to test samples, the duplicate test sample control chart can unquestionably monitor precision. By the use of a number of the above to monitor short term precision, limited use of control samples to monitor long-term precision, and occasional use of control reference samples to monitor for bias (particularly when developing a measurement process), the performance of the measurement process can be satisfactorily monitored.

All control charts, to be most effective, should be maintained in a real-time mode, to the extent possible. Responsive corrective actions then can be taken as necessary and questions of the validity of the data, due to out-ofcontrol operation, can be minimized. The control chart thus changes from a historical record (only useful if the system remains in control) to an active monitor of the measurement process, and provides the opportunity for feedback process control.

The question of the number of control charts to be maintained needs to be addressed. A practical answer should consider: the historical stability of the measurement process; the risk taken when the process is operated out-ofcontrol; and the variety of the work load.

Experience has shown that 5 to 10 percent of the sample load should consist of control samples of all kinds. More than this produces little extra information unless the system is excessively variable. In such a case, the causes should be sought and corrective actions taken to provide a more stable system. If less than the above percentage is analyzed, too high a risk factor may be involved. 
The kinds of control samples to be used have already been discussed briefly. Since they will be used to draw inferences about the measurement process, they should simulate the actual test samples or challenge the measurement process in the same way as the normal test samples. The professional judgement of competent analysts is required in making decisions as to the number and type of control charts and control samples that will be most beneficial in monitoring the measurement process.

In addition to control samples, control charts of other kinds may be found to be very beneficial. Examples of such include: calibration functions; extraction efficiencies; chemical blanks; and critical factors of a measurement process. When a critical factor is identified and monitored, measurement instability can be identified and corrected before the measurements themselves are affected. 
The term "sample" as used in chemical analysis denotes a portion of a material selected in some manner to represent a larger body of material. It is that portion, and sometimes a sub-portion (sub-sample) which is analyzed in order to draw conclusions about the body of material. Unfortunately, there are often sample-related uncertainties that can vitiate the conclusions or even prevent them.

Obviously, the selection of the sample is a critical operation. Questions of what samples to take, when, where, and how to take them, and how many to take and analyze are intimately related to the model (concept) of the problem and also depend on knowledge of the variance of the sample population and the variance of measurement. These questions are very important but are largely beyond the scope of the present document. Guidance for various aspects of sampling will be found in references $26,27,28,39,41$.

Questions concerning the integrity of samples will be discussed in the following sections.

\subsection{UNCERTAINTY OF THE SAMPLE}

The uncertainties of the sample may be classified into two categories: (a) those due to the natural variability of the sample; (b) those due to changes in the sample resulting from operations and conditions to which it may be subjected on removal from the population to which it belongs, during the course of analysis, and events that may occur in the ensuing interval.

The uncertainties of category (a) ordinarily must be evaluated and the measurement plan must be devised so that population variance and measurement variance may be de-coupled and estimated. The results of measurement should include estimates of uncertainty due to these two sources.

The uncertainties of category (b) are related directly to the quality assurance of the measurement process and fall into two groups, those due to sources of random error and those of a systematic nature. Even systematic errors can have a random component. For example, temperature may have a systematic (and even predictable and correctable) effect but its variability could produce random uncertainties. Factors which may affect the sample include: the sampling operation; contamination; degradation; interaction with. the container; mixing.

\subsection{QUALITY CONTROL OF SAMPLING}

The best way to deal with sources of sampling errors is to eliminate them or compensate for them to the extent possible. Often, this can be done by a well-designed and implemented quality control program. Just as in measurement, the use of GLP's, GMP's, and SOP's is the approach to quality control. PSP's for measurement programs should include protocols for all aspects of sampling, related to the specified measurement program.

In some cases, the quality control program for sampling can reduce sampling uncertainty to insignificant levels. When this is not achieved, estimates of the associated variances should be made and included in the total analytical uncertainty (see Appendix B). 
Because there are no "sampling reference materials", this approach to quality assessment is not possible. However, field blanks and known samples can be carried through various phases of sampling, including containment and storage, to monitor such operations. Monitoring of the cleaning operation for containers and of grinding and other kinds of sample preparation is possible. A valid and precisely implemented chain of custody can document that certain steps in a sample quality control program have been implemented.

\subsection{CHAIN-OF-CUSTODY}

The concept of "chain-of-custody" most often is viewed as a means for legal validation of samples, but its use for quality assurance is equally if not more important. An adequate system provides both assurance of identification of the samples that are analyzed and that all aspects of quality control required for them have been observed.

The simplest system for use in a chain-of-custody involves an appropriate label or tag that can be attached securely to the sample or its container. Such identifiers should be designed to trace the history of a sample, including the operations and conditions to which it has been subjected, and indicate places of residence together with times, dates, and the responsible individuals. The complexity of the system will depend upon the critical nature of the samples and the risk associated with the uncertainty of any significant information. In large and/or sensitive measurement programs, a sample custodian with responsibility for safeguarding samples and ensuring that the chain-of-custody is unbroken may be needed.

Whether or not a sample custodian is required, the management of samples can be a problem, even in small-scale operations. Often, samples must be subdivided when various parameters are measured by different analysts. Such sub-divisions must be made carefully to ensure the identity of sub-portions and without sample deterioration or introduction of contamination. In some cases, physical or chemical operations such as sieving and blending or dissolution and extraction are performed in a central location and the products are distributed to analysts. Obviously, these operations can be critical to the measurement data and must be subjected to appropriate quality control.

The use of dedicated facilities (such as clean rooms) and even reserved areas within them may be required to carry out sample-preparation operations. In ultra trace analysis, the hazard of cross-contamination by on-going samples and memory-effects of previous samples and even from laboratory reagents need to be considered and appropriate precautions must be exercised. Sample handlers must be thoroughly trained to perform their respective duties effectively and responsibly. 


\section{LEVELS OF QUALITY ASSURANCE}

Formally or informally, quality assurance is a necessary part of the production of data. No one will quarrel with the concept that measurements should be made with appropriate care and that limits of uncertainty are necessary for their logical use. Only the questions of how the goals are to be accomplished are debatable.

For complex operations, for those involving a number of participants, and for numerous repetitive measurements, a formal quality assurance program is virtually a necessity and the time and effort devoted to its development should be more than compensated by cost-effective and highly reliable analytical data.

Indeed, the cost of operation should not exceed 10 percent of the total operational effort and "do-overs" and "lost" data should be minimized. In production operations, it has been said that "quality is free" (11).

In research investigations, an informal quality assurance program may be adequate. However, the principles of quality control and quality assessment apply and their implementation, even in an informal way, could constitute a major portion of the experimental effort. Accordingly, research programs can formalize appropriate parts of their programs and substantially reduce the overall quality assurance effort in many cases. 
10. QUALITY ASSURANCE POLICY

The adoption and enunciation of a quality assurance policy is a prerequisite for an effective quality assurance program. This will reflect the degree of commitment of management to quality outputs and the resources that will be made available for this purpose. Based on the policy, a program can be developed and implemented. The policy should define the respective responsibilities of the various organizational levels in implementing the program. 


\section{PLANNING FOR QUALITY ASSURANCE}

Some degree of quality assurance is practiced in most laboratories. Often this is most of what is needed if the past measurement process has been well planned. Ordinarily, there is little opposition by laboratory personnel to doing a good job. However, there is a natural aversion to regulations and requirements, even when they define what is ordinarily done. When regulations include trivia or incredible requirements, they not only discredit the important ones, but engender contempt for the entire program. Accordingly, the quality assurance program must be both realistic and perceived as such.

The building blocks for a quality assurance program have been described earlier [51] and are illustrated in figure 5. The specificity increases in descending order. The policy of a laboratory reflects its basic measurement philosophy and the goals and objectives it desires to meet. It will reflect the type of service it strives to render and the desires and needs of the clientele' it serves. As already discussed, the GLPs and GMPs describe in rather specific terms the ways certain operations will be conducted. SOPs define how specific measurements will be made and the PSPs encompass all of the above for specific measurement activities.

A laboratory's quality assurance program should be internally motivated by the general desire to produce high quality outputs but it is externally motivated as well by specific requirements for a specific project. The former is thus program oriented and usually results in the development of a quality assurance manual. The latter is problem oriented and requires the development of project plans. The density of the tie-lines in the figure indicates the degree of relatedness of the central elements to the ongoing program or to a specific project.

A laboratory's quality assurance program, as delineated in its quality assurance manual, should be sufficiently comprehensive to define most of the measurement services which it can provide. Also, it may include PSPS for its recurring measurement activities. Ordinarily, PSPs will need to be developed for a specific project of a type not previously encountered, and especially for monitoring programs. Even when PSPs exist, they may need to be reexamined for their adequacy at each time of re-use. The figure suggests that, when a laboratory has a well-developed quality assurance program, PSP development can consist of the selection of elements appropriate to the project of concern with only minor modifications being required to adapt them to the specific program. 

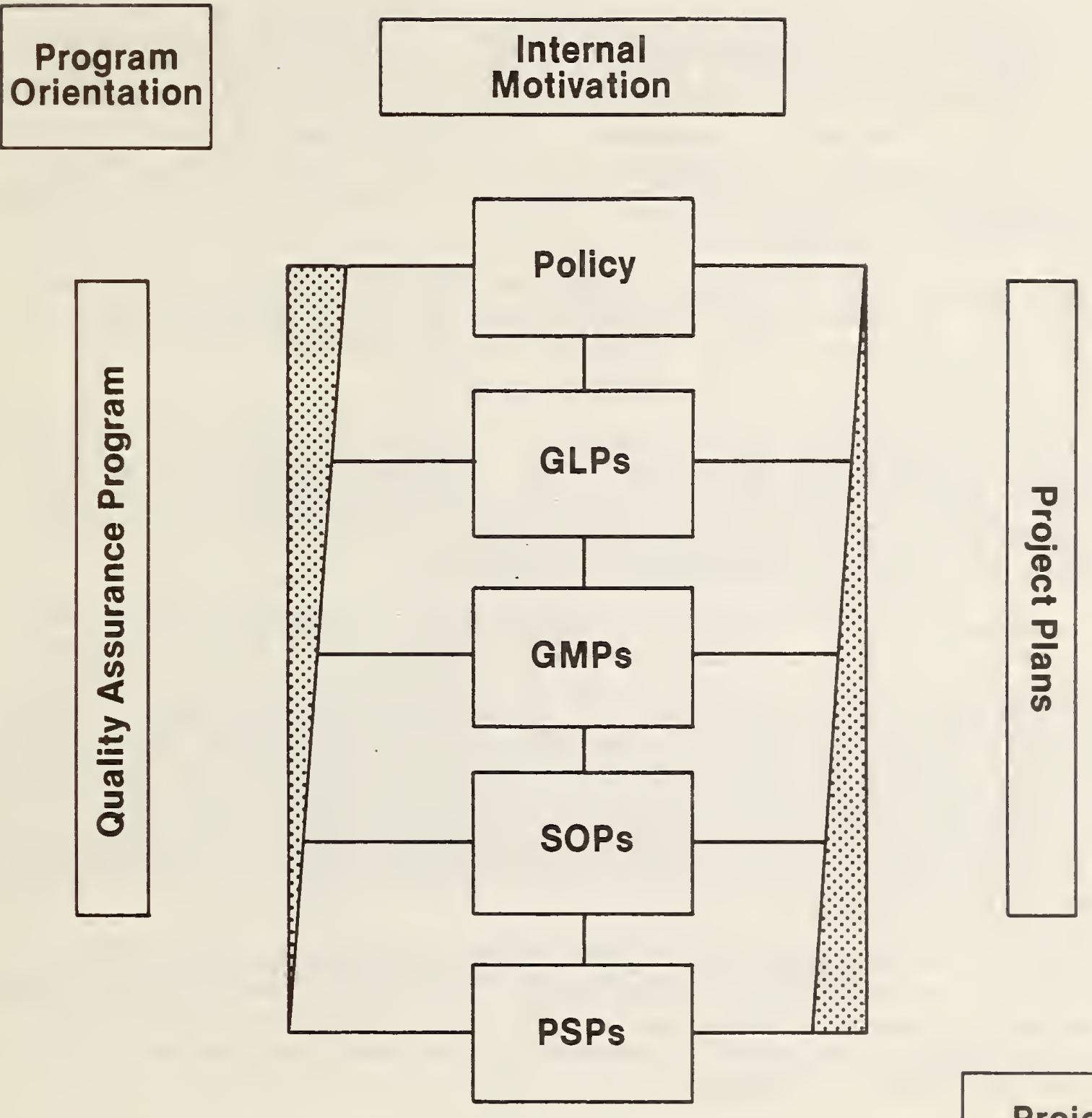

\section{Project Orientation}

\section{External}

Motivation

Figure 5. Quality Assurance Perspective 


\section{DEVELOPMENT OF A QA PROGRAM}

The development of a quality assurance program should involve all levels of laboratory personnel. The management must make the decision to develop a formal program, and establishes the policy that will be followed. In doing so, it should commit appropriate resources for both its development and implementation. Ordinarily, management designates a leader to develop the details of the program. Management will also need to approve the plans at various stages of its development and establish a mechanism for oversight and implementation of the program.

The leader may, or may not chair a committee, depending on the size of the organization. In either case, the leader must get the cooperation and involvement of the staff in developing the details and will follow appropriate procedures to obtain concensus approval.

The staff must provide technical advice and guidance, particularly in the development of the GLP's, GMP's, and SOP's. The staff may actually write appropriate parts of these documents or review what is written. Consensus approval of the staff is a necessity for general acceptability and for the preclusion of trivial and unnecessary requirements.

Staff involvement will be found to be educational for them and this may be one of the most important features of the development of a formal quality assurance program. The development process will require a careful consideration of the measurement process as it is practiced, internally, and as it is in the state-of-the-art. Parallelisms and contrasts between the practices of various groups and measurement techniques within a laboratory, can be identified, and the opportunity is provided to elevate all operations to the highest common factor. When the quality assurance program is assembled into the form of a manual, it can provide future guidance as well as documentation of present activities.

The process of development of the quality assurance programs need not be complicated. The developers should identify the goals to be accomplished and the motivation behind its development. This will include an examination of the internal policy and any $Q A$ requirements of external programs with which there is appreciable interaction.

The next step is to identify and document the quality assurance procedures already implemented in the on-going program. These should be compared with any requirements and also with the experience of others. Generic guidance is provided in the present paper and in several of the articles in the bibliography $[2,3,6,7,8,25,42]$.

The consensus approval, already referred to, is the next step. This will include concurrence by the internal participants of the program, by management, and by any external group or organization requiring a formal QA program, in the order mentioned.

Implementation of the quality assurance program is as important as its development. All levels of the laboratory staff are involved and the responsibilities of each should be clearly defined in the quality assurance program document. Management may designate a quality assurance officer or coordinator, to provide oversight of the program. The responsibilities of such an 
officer may range from periodic reviewing of the program and conducting systems audits to providing test materials and conducting internal performance audits on a routine and continuing basis. The review and approval of proposed work and development of the PSPs for such may also be a responsibility of this person/office. The quality assurance office should be independent of daily operations and report directly to management so that unbiased appraisals of operations can result.

Middle management (the supervisory staff) has the responsibility for direct implementation of the quality assurance program. Work of subordinates is reviewed for its technical excellence and conformance with quality assurance requirements. Middle management provides training related to both aspects and makes decisions on corrective actions, including the identification of assignable causes for measurement-process deviations.

The individual members of the technical staff have key responsibilities for the quality of the data output and for all related laboratory operations. The staff must be adequately trained to carry out both their technical and quality assurance responsibilities. Staff members often have the first opportunity to detect and correct malfunctions of the measurement system and must be trained to do this. Both the technical and supervisory staff must be able to distinguish between normal random fluctuations and abnormal deviations. Unnecessary over-corrections can often increase the variance of the measurement process and should be avoided.

The quality assurance program should be strictly enforced yet there should be flexibility to deviate from it as necessary. If deviations are necessary too frequently, the program may be defective and need to be amended. Deviation may be required due to both under- and over-specification of the quality assurance program or by changes in the measurement program that were not anticipated or are not covered by the program as originally developed. For such reasons the program should be continually reviewed and updated as required. While flexibility should be provided, the objective should be to develop a quality assurance document that can be followed with confidence and for which deviations would be exceptional and infrequent.

\section{QA PROGRAM DOCUMENTATION}

Should be clearly written

Should explain clearly what it is intended to accomplish

Should be accessible

Should be "user friendly" otherwise it will be ignored 


\title{
13. QUALITY ASSURANCE MANUAL
}

The quality assurance program and practices of a laboratory should be documented. Ordinarily they will take the form of a manual which will include:

The QA Program Document

\author{
GLPS \\ GMPs \\ SOPs \\ Implementation Directives
}

The quality assurance manual may be viewed as an instruction kit that the laboratory itself has developed to guide its laboratory operations in the production of quality data. It can provide internal guidance on policy and procedures and serve as a training manual for new employees. It should satisfy most external requirements, as well, so that its existence will minimize effort wherever PSP's need to be developed.

The quality assurance manual should be comprehensive but concise, and descriptive but brief. Because some parts will be general and others specific, it will need to be well organized and indexed. QA manuals may be made more useful when they are put on computers. Careful indexing can facilitate computer retrieval of information pertinent to a specific measurement problem, and thus enhance their usefulness. 
The general operational aspects of the quality assurance of a measurement process are shown in Figure 6 . Laboratory management is responsible for establishing policy and for managing both the technical operations and the quality assurance of the measurement program. The laboratory staff has the responsibility for conducting all operations and for their quality control in compliance with the associated GLPs, GMPs and SOPs. The staff provides also the major input to the development of the GLPS, GMPS, and SOPS. The protocols to be followed (PSPS) will depend on the nature of the problem to be solved and will encompass appropriate GLPS, GMPs and SOPS. Inputs from other sources such as the client, regulations, or specifications also influence the PSPS which will also contain the quality assessment procedures that are to be used.

In the problem solving mode, samples from the problem area are analyzed by technical operations that are in a state of statistical control, defined by the quality control program. The data output is screened for acceptability as defined by the PSP and released when found to be in conformance.

Figure 6 is not intended to be complete since some essential details have been omitted for simplicity. In fact, it should be considered in connection with figure 7 which depicts the overall process of analytical problem solving. This latter figure emphasizes the importance of an adequate model and of appropriate experimental plans in addition to the measurement aspects which constitute the bottom line.

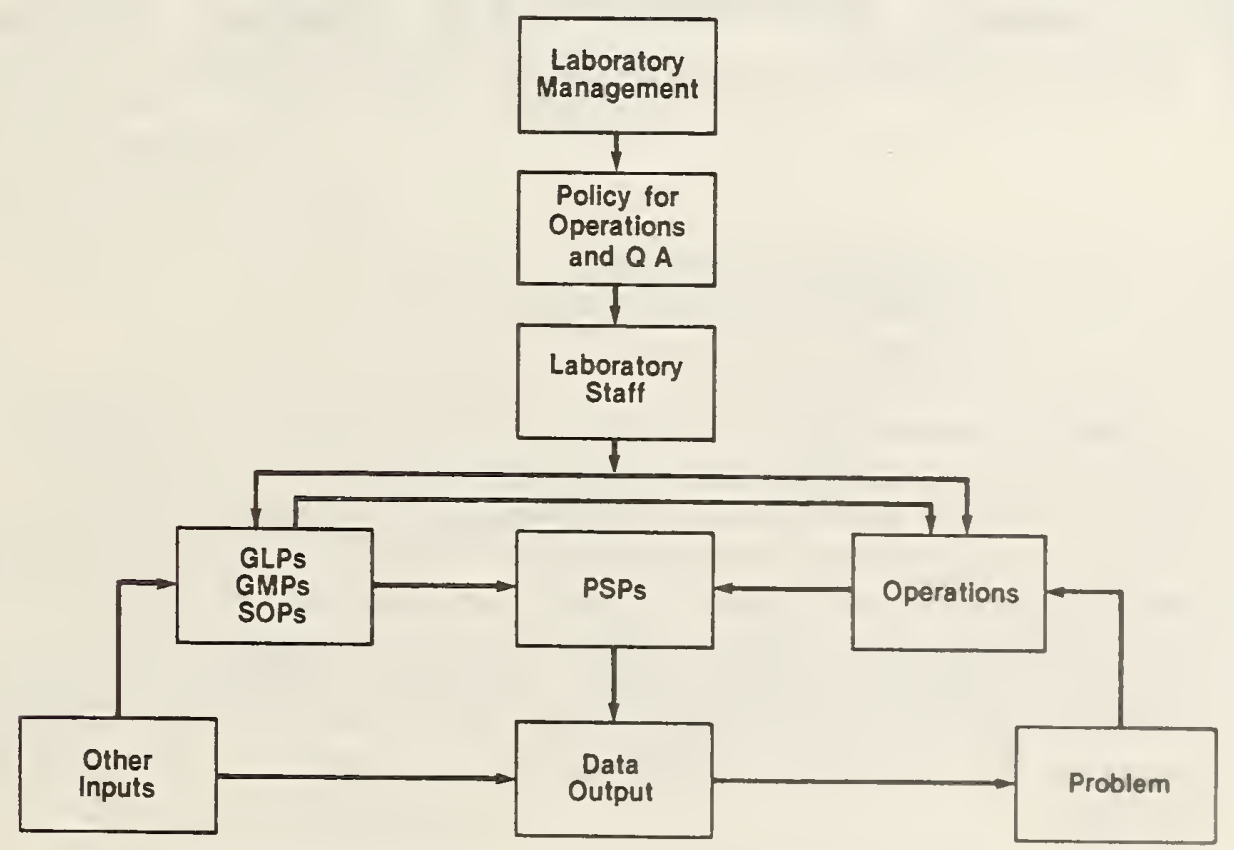

Figure 6. Quality Assurance of a Measurement Program 


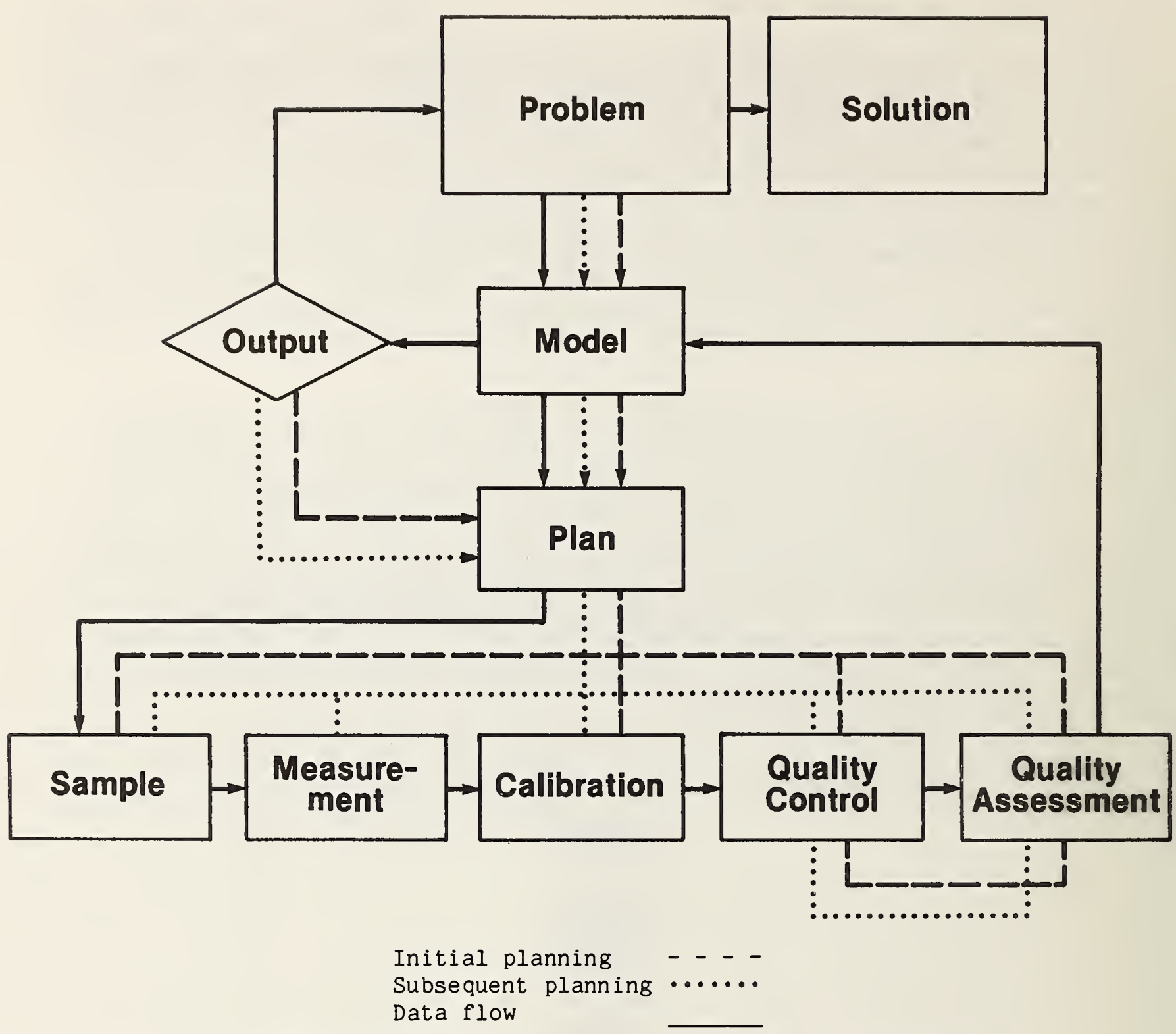

Eigure 7. The chemical measurement system. A problem is represented by a model which defines the data requirements and utilization. Based on the model, a measurement program may be planned which addresses sampling, calibration, methodology, and the quality assurance procedures to be utilized. The design and implementation of the chemical measurement system is often an interactive process and every measurement program should include a feed-back mechanism which provides for the identification of deficiencies and the initiation of corrective actions for all parts of the system, as required.

All components of the system are critically dependent upon each other and are implicitly or explicitly present in every analytical measurement activity. Failure to recognize this and to design the system properly is a common defect of many analytical programs. 
The PSP is the most specific aspect of a quality assurance program. It defines explicitly what is to be done in a given measurement situation. The sltuation may be as small as a simple analytical service offered by a laboratory or it may be as broad as an international monitoring program. The scope of a PSP is outlined in table 3.

The PSP is prepared by the authority responsible (or designee) for the measurement operation. This may be the laboratory director in the case of work to be done wholely within a single organization or the principal officlal of a monitoring program. When a PSP is developed for a particular recurring problem it becomes the way the pertinent data are to be obtained. When data are obtained for an external purpose, all interested parties may need to be involved in developing and/or approving the protocol. Needless to say, input should be sought at the bench level to make the protocol requirements meaningful and available.

The PSP is both a planning and an operational document and each aspect is a worthy reason for its careful preparation. It may be very brief with references to other documents as possible (and this is preferable) or it may be very detailed for major programs for which little precedent exists. The subjects to be addressed in the content are outlined in the following.

\subsection{DESIGNATION OF PRINCIPAL INVESTIGATOR}

The person(s) with overall responsibility for the implementation of the work should be designated together with the responsibilities that are delegated. A quality assurance officer should also be designated, as appropriate, together with the responsibilities thereof. Other subordinate responsibilities should be specified as appropriate, if not otherwise defined, such as in an organizational structure for example.

\subsection{DEFINITION OF PROBLEM}

The problem to be solved should be clearly defined. This will include a concise statement of the objective and the scope (limits) of the work to be undertaken. An overview of the activities may be included, as appropriate, with references to other related ones not included in the scope of the present project.

\subsection{SPECIFICATION OF THE MODEL}

The model refers to the concept of the problem, the assumptions that are made, and the hypotheses that are to be tested. The general data requirements are also set forth together with the precision and accuracy that should be achieved.

\subsection{SPECIFICATION OF SAMPLES}

This section will include details as to the number and kind (including location, time, etc.) of samples as required by the model. The methods of sampling, storage, preservation, subsampling, preparation for analysis and other pertinent information should be prescribed. The manner of distribution and chain-of-custody procedures may need to be specified in some cases. 


\subsection{SPECIFICATION OF DATA BASE}

This section will specify the data that should be obtained including that for analytical samples and for quality control and quality assessment. Measurement sequences may also be specified. The specification of the data base required to qualify a participant (or contractors) in a monitoring program as peers, prior to their participation, is a part of this section.

\subsection{SPECIFICATION OF METHODOLOGY}

The measurement methods to be used should be specified in sufficient detail so that no misunderstandings should arise as to what is to be done. Even when standard methods are specified, there may need to be additional information such as explanatory notes, for example. Any GLPs and GMPs, not given explicitly in the methods, but deemed as necessary to obtain measurements of requisite quality, should be included. Reference to appropriate GLPs and GMPs described elsewhere may be sufficient, but they should be readily available and reviewed for their applicability and adequacy in the present situation. Likewise, where deviations or exceptions from standard methodology are permitted, they should be indicated clearly.

\subsection{SPECIFICATION OF CONTROLS}

This section will describe all activities that will be undertaken to assure the quality of the data. Any necessary preliminary activities such as instrument warm up and performance checks should be described if not included in the SOP's. The corrective actions to be taken and the procedures used to indicate when these are necessary should be prescribed.

The control charts that should be maintained and the rationale for their interpretation should be specified. This will include those to be maintained by the measurement laboratory and those by others, such as a reference laboratory for example. All control charts should be kept on as close to a real-time mode as possible so that deviations from statistical control can be quickly detected.

The control samples to be used such as replicates, internal reference samples, Standard Reference Materials, blinds, and double blinds should be specified. In doing so, due consideration should be given to the model (to ensure its appropriateness) and the data base (to ensure its adequacy).

\subsection{RELEASE OF DATA}

This section should specify the requirements that the data must meet before it may be released by the originating laboratory and the criteria that the receiving organization will use to accept data. The persons authorized to judge the above should be designated also. When sensitive data are involved, the chain of official release should be defined. 
Table 3. Protocols for Specific Purposes*

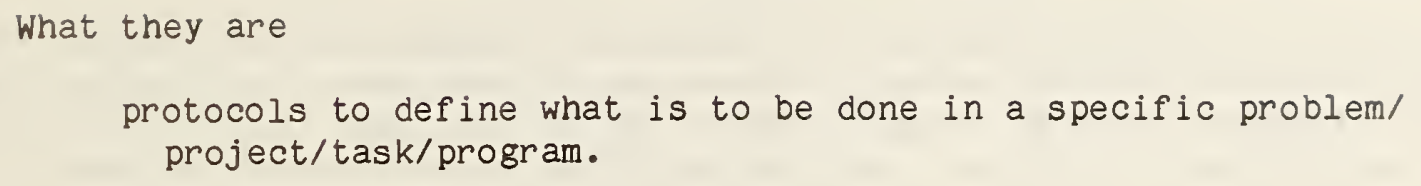

How prepared

by responsible authority

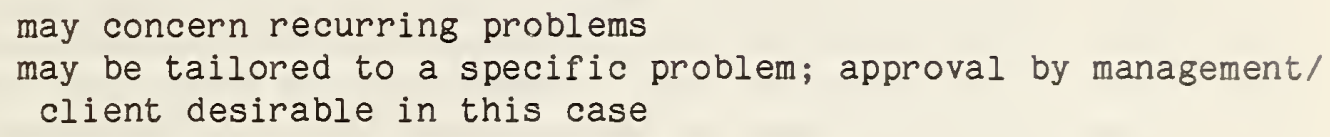

Content

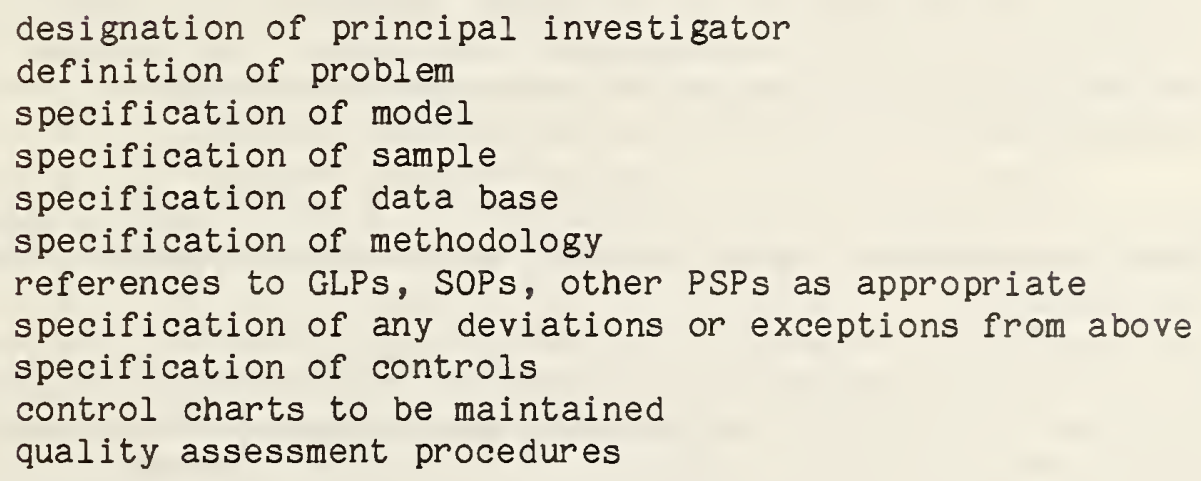

*Sometimes called project QA plans [45]. 


\section{QUALITY ASSURANCE FOR A MONITORING PROGRAM}

This section describes the quality assurance aspects of a monitoring program [51]. Monitoring programs may range in scope from those involving a number of participants over an extended period of time to individual laboratories seeking limited objectives. In any case, the program or project needs to be well planned and the data must be reliable if conclusions are to be drawn. The following discussion is written with large programs in mind but applies in principle to all such programs, regardless of size.

An adequate quality assurance program is a critical requirement for any monitoring program and the need increases with the number of participants and the complexity of the data base. Experience has shown that adequate quality assurance practices cannot be assumed, even for a single laboratory. By the laws of probability, the chance of faulty data will increase rapidly as the number of participants increases. Monitoring programs are often initiated in response to what is believed to be an urgent problem. The assumed urgency may override prudent planning in the haste to obtain information. However, if the planning and quality assurance procedures are faulty, the resulting data can be questionable, misleading, and even worthless.

The planning for any project and especially for a major monitoring program should proceed through the steps outlined in the following and illustated in figure 7 (page 30).

The problem which is intended to be solved by the monitoring data must be carefully defined. Precisely worded questions should be formulated that need quantitative answers. At the same time, the related questions which are not expected to be answered (that are not addressed) by the measurement program may need to be stated. In other words, there should be no uncertainty, at the start, as to what is hoped to be accomplished. This will not preclude inconclusive results but such, as they occur, should clarify the directions that should be pursued to obtain conclusive answers. In the case of a controversy, all parties concerned should agree that the questions are the right ones. Until such agreement is reached, it may be futile to obtain data since it will be impossible to reach any agreement as to what has been accomplished.

The model for the monitoring program also must be established in advance. This defines the data base as to kind and quality and specifies the samples that are to be analyzed. If comparisons are to be made with control sites, such comparisons should be based on samples in sufficent number, analyzed preferably by the participants, to make the results statistically interpretable. The model should describe how all data are to be reduced and evaluated.

Preliminary information ordinarily will be required to assist in framing appropriate questions and developing an adequate model. If such information is incomplete (and even if not) the questions and model will need to be re-examined, on the basis of initial data, to confirm their credibility, or to indicate revisions as necessary. In brief, the design of this as well as other parts of the program needs to be an iterative process and will need periodic re-examination to ensure its credibility and effectiveness. The worst thing that can be done is to hastily conceive a measurement program, blindly carry it out, and then try to make something of the data. 
The next logical step is planning. Based on the model, plans should be made for all aspects of the measurement process, including: sampling; measurement; calibration; and quality assurance. The PSP should be developed at this stage, however, this too should be an iterative process and revisions may be necessary, based on operating experience.

The PSP should clearly define what control charts are to be maintained and the requirements for demonstration of statistical control of the measurement process at the time that data are obtained for submission. The PSP should also address the subject of qualitative identification of the measurand and should state what confirmatory evidence is needed to support it.

When the limit of detection is of concern, the maximum acceptable value for the method limit of detection (MDL) (19) should be specified in the PSP and the frequency that the laboratory should confirm its MDL should be stated.

Because problem solving is essentially hypothesis testing, competent statistical assistance may be needed in problem definition, modeling, and planning. It is a common mistake to seek such advice after all data have been collected and attempts are being made to interpret them.

Intercalibration is an essential part of any multilaboratory monitoring program. That is to say, formal activities should be undertaken to ensure comparability and compatibility of the data produced. The first requirement is that the participating laboratories must have basic demonstrated competence in the specific measurement area (51). Then, they must maintain this competence throughout the program. Systems and performance audits before and during the program are essential. The latter need to be well planned with a rapid turn-around of the results so that appropriate corrective actions can be taken as they are required to eliminate measurement problems. The program authority has the responsibility to conduct the intercalibration program and it should provide technical assistance, as appropriate, to correct significant measurement problems. The use of a reference laboratory (see section 17 ) is an excellent way to accomplish this purpose.

An absolute requirement for all monitoring programs is peer review, prior to their initiation. At any time that major changes are required, peer concurrence should be obtained as well. All aspects of the program---the model, the plans, the measurement, the PSP's--should be scrutinized and all questions resolved before the program is activated. In the case of adversarial situations, all opposing factions should be represented in the review process.

A procedure similar to that known by the legal profession as "stipulation" may be followed to advantage in developing monitoring programs. In this, the attorneys involved agree in advance on how legal proceedings are to be conducted in a particular litigation. Similarly, in monitoring programs, all parties concerned should have the opportunity to agree on what is to be done also (what cannot be done in some cases) and on any limitations based on such considerations as time, cost, availability of samples, and the state-of-theart of measurement and interpretation of the data. Unless there can be a reasonable consensus on such matters, in advance, the outcome of the work. even when properly done, can be disappointing and raise questions ranging from minor to major. In the case of the latter, one should ask whether the 
proposed work is worthwhile and whether research or effort should be devoted to delineate the problem and methods for its solution, before a monitoring program is activated.

A monitoring program must recognize that quality of data is a paramount consideration and must devote sufficient resources to accomplish this goal. When purchasing measurement services, realistic quality performance specifications and quality assurance practices should be established and vendors should be held accountable for following them. Laboratories should not be paid for data of insufficient or undocumented quality [51]. 


\section{FUNCTIONS OF A REFERENCE LABORATORY}

Large monitoring programs may find it advantageous to use a reference laboratory (RL) to provide services which would increase confidence in the data, intercalibrate the measurement laboratories within the network, maintain continuous surveillance of the measurement program, and advise on corrective actions as required. The reference laboratory may provide a gamut of services ranging from advice to full responsibility for operation of a monitoring program. The reference laboratory concept is presented in outline form in appendix A.5 and is summarized in the following.

Several possible interrelationships of a reference laboratory to others in a monitoring program are illustrated in figure 8. Obviously, the intercalibration lines must be strong if all data are to have peer status and are to be compatible. The strength of the other bonds will depend on the degree of responsibility delegated to the reference laboratory. The latter may have full responsibility for the entire program, in which case the direct lines between the user and the measurement laboratories could be virtually non existent. When the user assumes major management responsibilities, the lines between the reference laboratory and the measurement laboratories accordingly will be weaker.

An effective reference laboratory should have the following qualifications. It should have recognized competence and a dedication to high quality work. Preferably, it should be a recognized center of excellence with broad interdisciplinary capability and experience. It should have a reputation for impartiality and objectivity. Furthermore, and as a major requirement, it must have an effective ongoing quality assurance program to serve as a model for the measurement laboratories, to back-up its technical opinions, and to provide authoritative support for its own data outputs.

A well-qualified reference laboratory can provide some or all of the following functions to a monitoring program (see table 4). It may provide research and development support with respect to both methodology and reference materials. With respect to the former, it may validate proposed methodology for use in the monitoring program, identify gaps in the measurement process, and develop new or improve exisiting methods as needed. It may conduct collaborative tests to do some of the above. Concerning reference materials, it can investigate various options and validate and recommend materials for uses ranging from routine measurement control to proficiency testing of participating laboratories. Again, when suitable reference materials are not available, the reference laboratory may be able to develop ones appropriate for the specific monitoring program.

The reference laboratory may serve in a referee laboratory capacity. As such, it can analyze (or re-analyze) a fraction of the total samples to verify measurement consistency and to corroborate key data to increase confidence in it. The reference laboratory may engage in trouble shooting, as necessary, with respect to sampling, methodology, or other aspects of the program. 
Table 4. Functions of a Reference Laboratory in National Measurement/Monitoring Programs

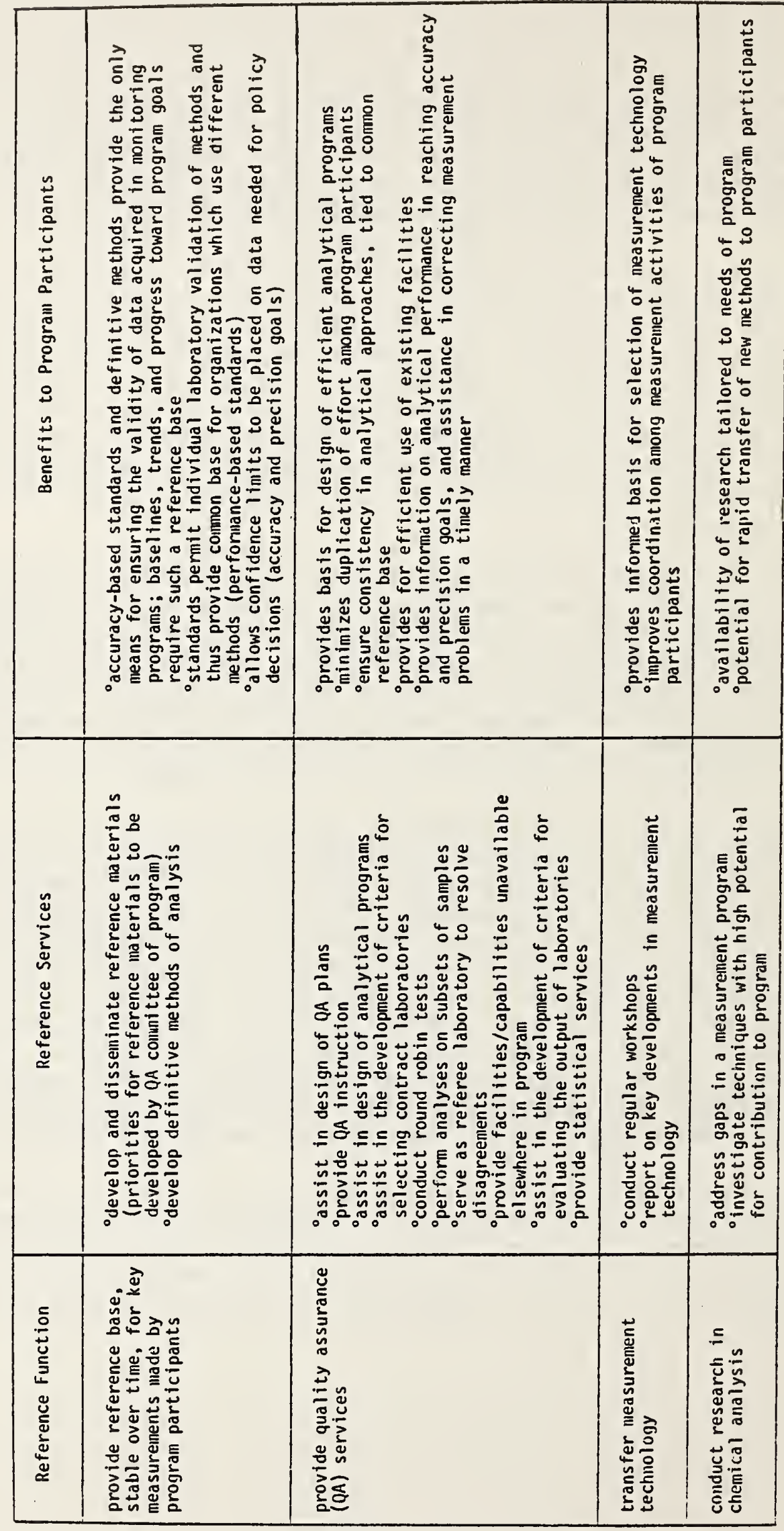




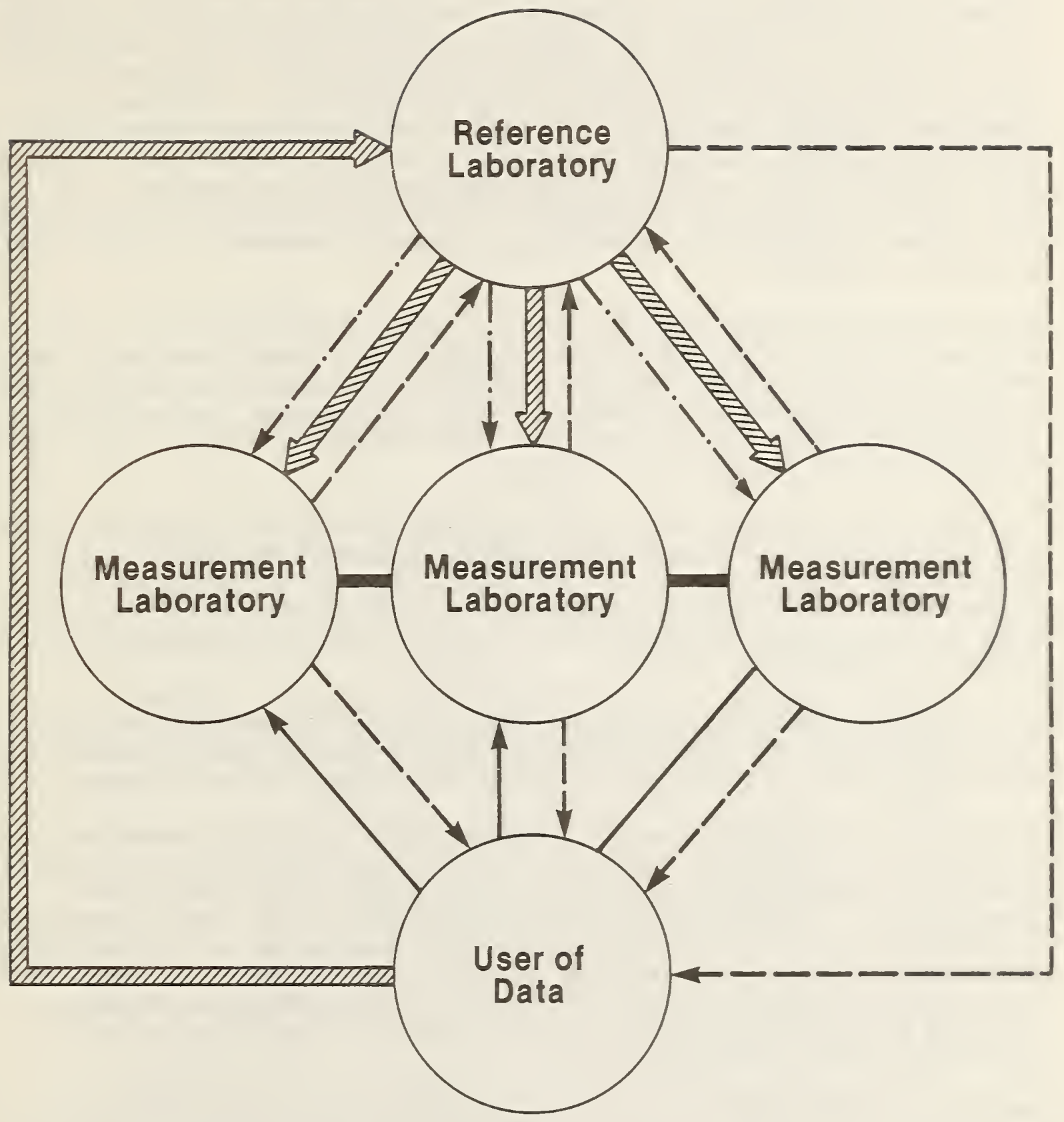

Sample Flow, Distribution by User

- Data Flow

-.- QA Sample Flow

ZImIIII Alternate Sample Flow, Distribution by Ref. Lab.

Intercalibration Program

Figure 8. Cooperative Measurement Program 
The quality assurance function can range from furnishing advice to oversight of the entire activity (see figure 9). The reference laboratory may be used to review and pre-validate the quality assurance program and to demonstrate that it is credible and can be expected to be effective. Likewise the PSP for the entire monitoring program can be designed and/or validated by a competent reference laboratory. It may conduct proficiency tests of the measurement laboratories including those to qualify potential participants and to verify their continuing competence throughout the program. The circulation of samples and evaluation of inter-calibration test results can be a prime responsibility. Relatedly but possibly separately, it may provide statistical services.

The reference laboratory may be given a training responsibility. It is recommended that larger monitoring programs should conduct workshops, before and at intervals during a monitoring program, to indoctrinate participants, to maintain awareness of programmatic goals and progress, and to introduce any necessary changes in the program. The reference laboratory could assume responsibilities for such activities.

The reference laboratory and/or the user should maintain a continuous alert for deficiencies in the monitoring program that would be correctible by training and provide such on an individual laboratory or group basis as appropriate. Such training conceivably could be in the use of the methodology for measurement and sampling or in quality assurance aspects of the monitoring program.

In a continuing monitoring program, the reference laboratory also could have the responsiblity to groom a successor or to develop a line of succession, as described below, to provide for internal surveillance of the program.

Because of its important function, the reference laboratory should be selected with great care. It may be chosen from laboratories not regularly engaged in the monitoring program or it may be one of the measurement laboratories. The former approach is recommended in the case of extensive programs and the latter is possible in small programs or as a mechanism for maintaining a larger program after it is well established. In such a case, the reference laboratory could be given the responsibility to develop a training program that would provide for internal surveillance by the participants on a rotational basis.

The initial selection of a reference laboratory and of any internal successors is based on finding a laboratory with unquestionable qualifications and this must receive consensus approval. The reference laboratory must maintain a high degree of credibility throughout its tenure since it will be quite influential in developing and implementing the monitoring policy. 


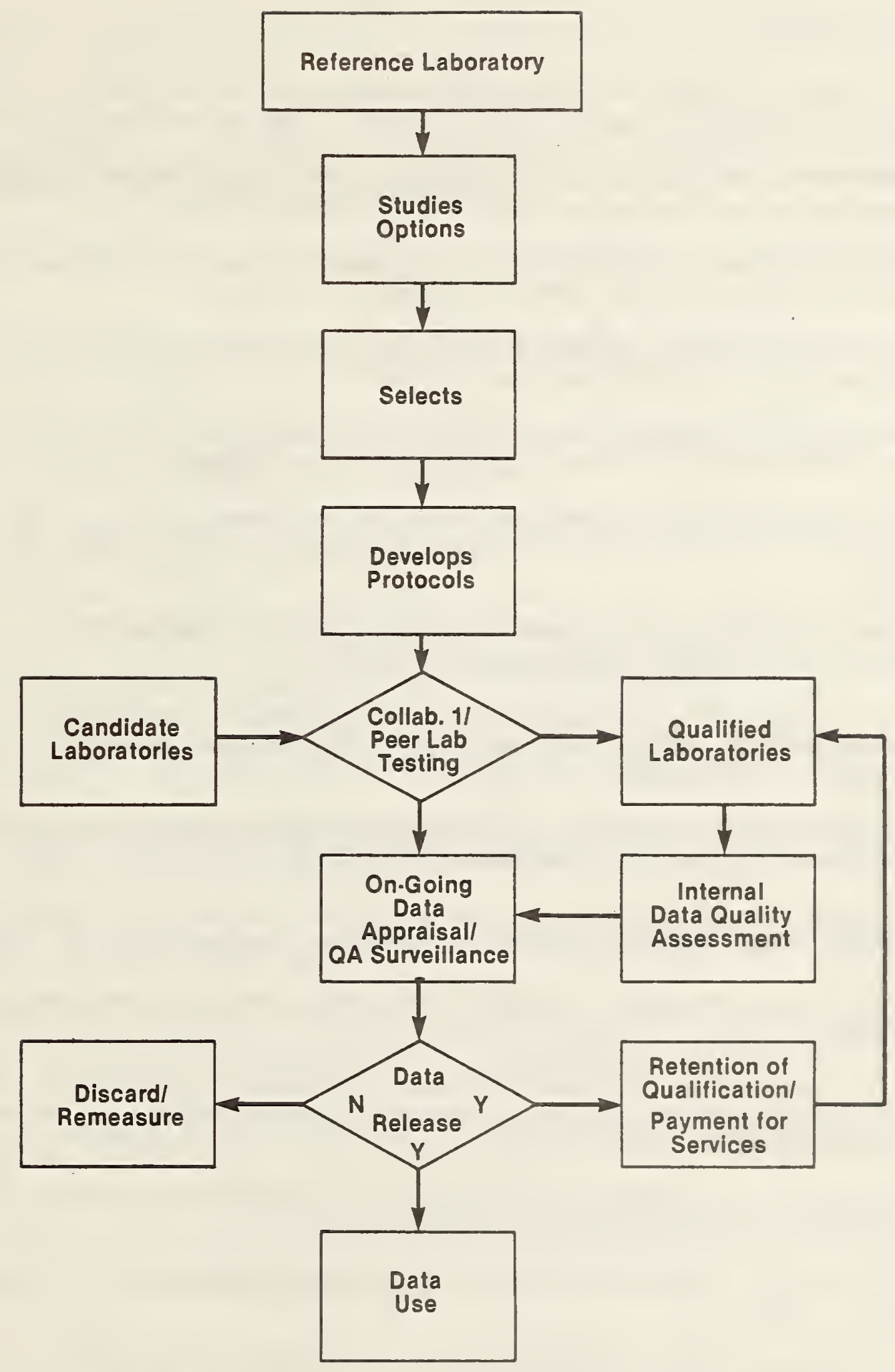

$\therefore$ igure 9. Quality Assurance Function of a Reference Laboratory 
18. BIBLIOGRAPHY

1. ASTM D2777, Standard Practice for Determination of the Precision and Bias of Methods of Committee D19 on Water, ASTM Philadelphia, PA 19103.

2. ASTM D3614, Evaluating Laboratories Engaged in Sampling and Analysis of Atmospheres and Emissions, ASTM Philadelphia, PA 19103.

3. ASTM D3856, Evaluating Laboratories Engaged in Sampling and Analysis of Water and Waste-Waters, ASTM Philadelphia, PA 19103.

4. ASTM E178, Standard Recommended Practice for Dealing with Outlying Observations, ASTM Philadephia, PA 19103.

5. ASTM E305-83, Standard Practice for Establishing and Controlling Spectrochemical Analytical Curves, ASTM Philadelphia, PA 19103.

6. ASTM E-548, Standard Recommended Practice for Generic Criteria for Use in Evaluation of Testing and/or Inspection Agencies, ASTM Philadelphia, PA 19103.

7. ASTM E-748, Quality Assurance Procedures for Spectrographic Laboratories, ASTM Philadelphia, PA 19103.

8. G. A. Berman, Ed., Testing Laboratory Performance: Evaluation and Accreditation, NBS Special Publication 591, National Bureau of Standards, Gaithersburg, MD 20899.

9. R. Bordner and J. Winter, Microbiological Methods for Monitoring the Environment, Water, and Wastewater, EPA-600/8-78-017, Dec. 1978.

10. J. Bryson, et al, Bibliography on Laboratory Accreditation, NBSIR 82-2523 (1982) National Bureau of Standards, Gaithersburg, MD 20899.

11. P. B. Crosby, "Quality is Free", McGraw-Hill Book Co., New York, NY (1979).

12. J. P. Cali, et al, The Role of Standard Reference Materials in Measurement Systems, NBS Monograph 148, National Bureau of Standards, Gaithersburg, MD 20899.

13. ASTM Manual on Presentation of Data and Control Chart Analysis, STP 15D, ASTM, Philadelphia, PA 19103.

14. W. E. Deming, On Statistical Aids to Economic Production, Interfaces August, 1975, p 5.

15. C. Eisenhart, Realistic Evaluation of the Precision and Accuracy of Instrument Calibration Systems, In Ref. 26, pp 21-47.

16. J. J. Filliben, Testing Basic Assumptions in the Measurement Process, in "Validation of the Measurement Process", J. R. DeVoe Ed., ACS Symposium Series No. 63 (1977), American Chemical Society, Washington, DC 20036. 
17. L. C. Friedman and D. E. Erdmann, Quality Assurance Practices for the Chemical and Biological Analyses of Water and Fluvial Sediments-Chapter A6 in "Techniques of Water-Resources Investigations of USGS" (1982). U.S. Government Printing office, Washington, DC 20402.

18. S. Gaft and F. D. Richards, Quality Assurance at Ford Motor Company Central Laboratory-A dynamic Approach to Laboratory Quality, in ASTM STP/814, ASTM Philadelphia, PA 19103.

19. J. A. Glaser et al. Trace Analysis for Wastewaters - Method Detection Limit, E. S. \& T. 15, 1426 (1981).

20. Guidelines for Data Acquisition and Data Quality Evaluation in Environmental Chemistry, American Chemical Society, Analytical Chemistry 52: $2242 ;(1980)$.

21. Handbook for Analytical Quality Control in Water and Wastewater Laboratories EPA 600/4-79-019, March 1979.

22. C. D. Hendrix, What Every Technologist Should Know about Experimental Design, CHEMTECH, March 1979, p. 167.

23. H. S. Hertz and S. N. Chesler, Eds. Trace Organic Analysis: A New Frontier in Analytical Chemistry, NBS Special Publication 519 (1979). National Bureau of Standards, Gaithersburg, MD 20899.

24. J. Stuart Hunter, Calibration and the Straight Line: Current Statistical Practices, AOAC Journal 64, 574 (1982).

25. ISO Guide 25, Guidelines for Assessing the Technical Competence of Testing Laboratories, American National Standards Institute, 1430 Broadway, New York, NY 10018.

26. B. G. Kratochvil and J. K. Taylor, Sampling for Chemical Analysis, Anal: Chem. 53, $924 \mathrm{~A}$ (1981).

27. B. G. Kratochvil and J. K. Taylor, A Survey of the Recent Literature on Sampling for Chemical Analysis, NBS Technical Note 1153, January 1982, National Bureau of Standards, Gaithersburg, MD 20899.

28. B. G. Kratochvil and D. Wallace, and J. K. Taylor, Sampling for Chemical Analysis, Anal. Chem. Reviews, 56, 113R.

29. H. S. Ku, Editor, Precision Measurement and Calibration: Statistical Concepts and Procedures, NBS Special Publication 300, Vol. 1, Stock No. 003-003-00072-8 Superintendent of Documents, U.S. Govt. Printing office, Washington, D.C. $20402(\$ 11.00)$.

30. P. D. LaFleur, Ed., Accuracy in Trace Analysis: Sampling, Sample Handling, Analysis, NBS Special Publication 422 (1976), National Bureau of Standards, Gaithersburg, MD 20899.

31. J. Mandel and T. W. Lashof - Interpretation and Generalization of Youden's Two-Sample Diagram, J. Quality Technology, 6, 22-36 (1974). 
32. A. G. McNish, Dimensions, Units, and Standards, Physics Today, 10, 19-25, April 1957.

33. W. W. Meinke, Ed., Trace Characterization, Chemical and Physical, NBS Monograph 100 (1967), National Bureau of Standards, Gaithersburg, MD 20899.

34. M. G. Natrella, Experimental Statistics, NBS Handbook 91, Stock No. 003-003-00135-0, Superintendent of Documents, U.S. Govt. Printing office, Washington, D.C. $20402(\$ 18.00)$.

35. L. S. Nelson, Use of Range to Estimate Variability, J. Qual. Technology, I. 46 (1975).

36. W. Nelson, How to Analyze Data with Simple Plots, ASQC Basic References in Quality Control - Statistical Techniques, American Society for Quality Control, Milwaukee, WI 53203.

37. Applied Linear Statistical Models, J. Nether and W. Wasserman, p. 140-145 (1974), Published by Richard D. Irwin, Inc., Homewood, Illinois 60430 .

38. W. E. Oatess - Establishment of Accreditation Programs for Environmental Labs, Env. Sci. Tech. 12; 1124 (1978).

39. L. P. Provost, Statistical Methods in Environmental Sampling, ASCE Symposium Series No. 267, American Chemical Society, Washington, DC 20036.

40. Precision Measurement and Calibration, NBS Handbook 77, National Bureau of Standards, Gaithersburg, MD 20899.

41. Principles of Environmental Measurement, American Chemical Society, Anal. Chem. 55: 2210 (1983).

42. Quality Control System - Requirements for a Testing and Inspection Laboratory, American Council of Independent Laboratories, $1725 \mathrm{~K}$ Street N.W., Washington, D.C. 20006.

43. Standard Reference Materials and Meaningful Measurement, NBS Special Publication 408, National Bureau of Standards, Gaithersburg, MD 20899.

44. Standard Reference Materials Catalogue, NBS Special Publication 260. National Bureau of Standards, Gaithersburg, MD 20899.

45. T. W. Stanley and S. V. Verner, Interim Guidelines for Quality Assurance Project Plans, NTIS-PB 83-170514 (1983).

46. J. K. Taylor, Importance of Inter-Calibration in Marine Analysis, Thal. Jugo. 14, 221 (1978).

47. J. K. Taylor, Quality Assurance of Chemical Measurements, Anal. Chem. 53, 1588A (1981). 
48. J. K. Taylor, Quality Assurance Measures for Environmental Data, in "Lead in the Marine Environment", M. Branica and Z. Konrad Eds., Pergamon Press (1980) p. 1.

49. J. K. Taylor, Validation of Analytical Methods, Anal. Chem. 55, 600A (1983).

50. J. K. Taylor, Reference Materials - What They Are and How They Should be Used, J. Testing and Evaluation 11, 355 (1983).

51. J. K. Taylor, Quality Assurance for a Measurement Program, ACS Symposium Series No. 267, American Chemical Society, Washington, DC 20036.

52. J. K. Taylor, Essential Features of a Laboratory Quality Ássurance Program, in Statistics in Environmental Sciences, ASTM STP 845, ASTM Philadelphia, PA 19103.

53. G. Wernimount - Ruggedness Evaluation of Test Procedures, ASTM Standardization News, March 1977, p. 13.

54. J. 0. Westgard and T. Groth, A multi-rule Shewhart Chart for Quality Control in Clinical Chemistry, Clinical Chemistry 27, 493 (1981).

55. William J. Youden, Collection of Papers on Statistical Treatment of Data, Journal of Quality Technology, $\underline{4}, 1$ (1972).

56. Ranking Laboratories by Round Robin Tests, W. J. Youden, Materials Research and Standards, January 1963.

57. W. J. Youden and E. H. Steiner, Statistical Manual of the Association of Official Analytical Chemists, AOAC, 1111 North 19th Street, Suite 210 , Arlington, VA 22209. 


\section{INDEX TO BIBLIOGRAPHY}

Accreditation

$$
8,10,38
$$

Calibration

$15,24,40,46$

General

$15,20,23,30,32,33,41,45$

Methodology

$1,5,9,15,17,19,23,30,33,49$

Quality Assurance Systems

$2,3,6,7,9,13,17,18,21,25,38,42,45,46,47,48,52,53$

Samples, Sampling

$26,27,28,39$

Standard Reference Materials

$12,43,44,50$

Statistics

$1,4,5,13,14,22,29,31,34,35,36,37,3951,52,53$, $54,55,56,57$ 
This appendix contains supplemental material which may be useful in the development of documents related to the quality assurance program of a specific laboratory. The material is generic in content and is believed to be widely useful. However, each laboratory should consider its applicability and will need to develop the specific content most useful to its own measurement program.

The material in this appendix consists largely of outlines that can be followed when preparing specific documentation. It is recommended that a laboratory or organization which uses such should address each item in an outline, and prepare text as appropriate for its own situation.

An exception to the outline format is Appendix A.1 which contains the text of a program document that one laboratory has found to be appropriate to its measurement situtation. This document is presented as an example of such a document and is presented only for guidance.

\section{A. 1 EXAMPLE QUALITY ASSURANCE PROGRAM DOCUMENT}

1. Policy

The objective of the measurement program of the

$$
\text { is to produce high-quality research and }
$$

analytical measurements and data which are accurate, reliable, and adequate for the intended purpose in a cost-effective manner. To this end, the management is dedicated to the encouragement of excellence in measurement and to provide the physical and mental environment conducive to its achievement.

\section{Purpose}

The purpose of this document is to formalize the quality assurance practices that have evolved during the past and which are currently operative within the A further purpose is to inform users of the services of the conditions and practices related to the services and data provided.

Due to the diversity of programs, it may not be possible to set forth all inclusive statements nor to establish a rigid quality assurance plan. The management encourages the developement and use of the best measurement practices as dictated by each measurement situation. However, it is the policy to follow the procedures set forth herein to the extent possible and to document the experimental details in each individual case and maintain them as a matter of record.

\section{General Aspects}

\subsection{Preliminary Requirements}

All analytical measurements will be done according to a plan individually developed and optimized for each situation. The requirements of each problem will be studied and thoroughly understood beforehand, in order to assure that the measurements planned will be adequate both in kind, number and quallty. All measurements are made using standard methods, methods with peer 
recognition, methods developed in the specific laboratory, or those mandated by legal or other requirements. No method will be used for data output purposes until it is known to be applicable and competence has been acquired in its use. If or when it appears that available techniques are not adequate to solve a particular problem, the user of the services will be informed of any preliminary investigations or research required, including the estimated costs.

Demonstration and documentation of attainment of statistical control of the measurement process shall be a prerequisite for reporting any data, whether of a research or routine nature.

All laboratory equipment shall be maintained in a reasonable and proper state of repair. Space shall be maintained according to established rules of cleanliness, order, and efficiency to facilitate the measurements and to protect the health and safety of the laboratory personnel. No equipment is operated until it is first ascertained that it is in a safe and reliable state and then only by personnel who have been thoroughly trained and have been duly qualified as operators.

Work done for another organization will adhere to any specified project plan or quality assurance program to the extent possible. Whenever such directives differ from on-going policy, such differences will be reconciled before work is initiated or whenever they are first recognized.

\subsection{Experimental Plan}

All experimental work is to be done according to a written plan developed in advance of the actual work. Where applicable and appropriate, the plan is developed in consultation with the end-user of the analytical data and the statistician who will assist in interpreting the data or reviewing the conclusions. The plan includes the sampling strategy, the sampling procedure, the calibration process, the measurement procedure, and the data handling and validation process. All procedures used should be documented and the documentation should be available for review by users of the data. Standard Operations Procedures (SOP's) are used to the extent practical. When other procedures are required, their adequacy is verified before use in a measurement process.

\subsection{Reagents}

Reagents are defined as any substances used to dissolve, disperse, extract, react with, or dilute any sample or analytical component of a sample. Strict control of reagents shall be maintained to minimize their contamination or degradation. Dilutions or solutions prepared from such reagents shall be clearly labeled and dated. The shelf life shall be designated and strictly observed.

The highest quality analytical reagents, consistent with the end use, shall be used in all analytical work. Records will be kept of all reagents used in every critical analysis and each analytical plan shall include procedures to carefully control the quantities used and to minimize and correct for the analytical blank to the extent possible. 
The policy of the

is as follows: General safety rules as developed by will be followed. Special safety rules in addion to the above will be part of any GMP for a specific measurement competence.

\subsection{Disposal of Toxic Substances}

The potential hazard of each substance used regularly or occasionally will be investigated as a prerequisite for its use. No potentially hazardous material will be used until it has been approved for use by at least one level of supervision above the user. The Safety office will be consulted in each case as appropriate and disposal will be by that office or in accordance with its approved procedures.

4. Samples

It is recognized that sampling is one of the most critical operations in analytical measurement. The analyst can only report on the measurement made on a specific sample at a specific time. Often this is not the information ultimately desired but rather the projection of that information in time and space and its relation to the population from which the sample was drawn.

The sampling strategy, methodology, subsampling, chemical and/or physical treatment, preservation, and storage are logically parts of the analytical plan and must be developed and specified for each situation.

Sampling plans and measurement procedures must be adequate to permit evaluation of sample homogeneity and/or population distributions when these are important considerations.

Any limitations on the analytical results due to the sample must be clearly specified in the analytical report. It is the policy of the that the integrity of samples should be unquestionable. All samples are to be identified in an unambiguous manner and fully documented in the respective record books. Samples shall be stored to minimize contamination and/or deterioration while in the and shall be retained as long as necessary in the judgement of the supervisor or delegated authority. Adequate records shall be kept to establish a chain of custody and to ensure their integrity, identification, and locatability at all times. When necessary or mandated, a legal chain-of-custody shall be established including the designation of a sample custodian.

\section{Analytical Methodology}

\subsection{General}

All analytical measurements shall be made using methodology appropriate for the specific purpose. Each measurement method will be described in writing before use in the generation of analytical data. This serves the purpose of recording the procedure used and also ensures that a sequence of measurements will be made using the same procedure, which is a prerequisite for statist1cal treatment of the resulting data. Any significant changes in a measurement procedure shall be documented and notebook reference shall make clear when such changes are made. 
When changes are made to an SOP, the specif1c changes, together with the rationale for them, shall be documented and clearly indicated whether the change is permanent or pertains to a speclallzed use. Any signiflcant changes will be considered as generating a new method which will need to be validated before 1 t may be used for definitive measurements.

The written procedure will include the following information, as applicable:

a. Sample preparation and treatment

b. Chemical operations

c. Calibration procedure

d. Measurement procedure

1. Instrumental conditions

2. Instrumental adjustments

e. Data handling (See 4.3 )

References to Standard Operations Procedures (SOP'S) when they are used will satisfy this requirement for documentation.

\subsection{Standard Operations Procedures (SOPs)}

Standard operations procedures (SOPS) designate those measurement procedures or sub-steps which have been established by consensus actions of standards organizations or those reduced to a standard format and demonstrated by consistent use in the laboratory to be the preferred way to do a measurement or operation of concern.

Measurement procedures or subsets thereof which are used in a recurring manner should be written as SOPs to which reference can be made in the event of future use.

SOPs will be written in a standard format when possible. The format of preference is that used by ASTM.

\subsection{Calibration}

5.3.1 Calibration generally refers to physical measurements made on objects, apparatus, or equipment to verify or provide corrections to the experimental data it provides. Examples include masses, volumetric apparatus, and flowmeters. Records of such calibrations, whether made externally or internally, will be maintained in a central location within the corresponding division. Copies are also made avallable to the users of the calibrated articles and supervisors will be responsible to see that the latest calibration information is applied in each pertinent situation.

5.3.2 Standardization is a type of chemical calibration and refers to those operations in which instrument response (in its broadest interpretation) is related to analytical data. Examples include the titer of a solution, an absorbance-concentration curve, and the spanning of a linear-direct reading chemical analyzer. Except for the latter, such data are ordinarily recorded in notebooks. The reliability of many measurements is critically dependent on the quality of the chemical calibrants used in the standardization 
process. This may involve obtaining primary standards and the preparation of secondary standards. In such cases, labeling, record of preparation, expiration date, and special precautions for use are prime considerations. In some cases, calibrants may be obtained from external sources such as a gas manufacturer.

5.3.3 It is the responsibility of each analyst to see that the concentrations of all chemical standards used are verified by reliable methods. The procedures used for such verification, as well as the use of standards shall be fully documented.

5.3.4 Calibrations and standardizations shall be made with sufficient frequency to minimize uncertainties in the analytical results due to uncertainties of calibration and/or standardization.

5.3.5 All calibrations and standardizations shall be fully documented and the data shall provide clear traceability to the calibrations related thereto.

\subsection{Data Handling}

Descriptions of each analytical method shall include procedures for handling data. Because the procedures may be common to many of the measurements in a given laboratory, this may be best described as a GLP. The following subjects should be addressed, as appropriate:

- Procedures used in the calculation of data; corrections required; adjustment to standard conditions; normalization of data; computer programs.

- Statistical procedures used to report data; method for evaluating limits of uncertainty.

- Corrections for sytematic errors.

- Source of all constants used in calculations, such as Faraday constant, atomic weights, molecular weights.

- Checks on accuracy of calculations; use of significant figures; propagation of errors; analysis of variance.

6. Laboratory Records

\subsection{General}

It is the responsibility of each individual in the complete records of her/his work in the detail and format prescribed by the particular organizational unit. The minimum requirement shall be that all significant experimental work shall be described in sufficient detail to make it understandable to one versed in the art, yet not directly connected with the work.

All laboratory record books are the property of the and may not be removed from the premises without permission of higher author 1ty. Records are to be suitably protected and stored to conform with the general regulations of the - Individuals who wish to maintain personal records may do so, but these shall be in addition to the complete and permanent records mentioned above. 


\subsection{Measurement Data}

All experimental data will be recorded in bound laboratory record books. Transcriptions shall be avoided wherever possible. The data shall be suitably annotated with reference to experimental details, conclusions and/or future directions as needed to positively identify it. The data record may be kept in a general laboratory record book or in a separate book, as approved by supervisors, but cross references shall be made as appropriate. Corrections to recorded data shall be documented and no erasures will be permitted. The name of the analyst shall be clearly indicated so that there is no question as to who is responsible for the work.

Data in the form of charts, instrument recordings, and printouts will be given suitable identification numbers and maintained in a manner consistent with that indicated for written records. Laboratory record books will refer to the location and identification number of such records.

In view of the possible legal implications of much of the data, all data shall be recorded in such a way as to preclude its discreditation at any time. Any data obtained for use of patent applications or for other legal purposes may need to be substantiated by witnesses. The rules promulgated by the legal advisor will be followed in such cases.

\subsection{Maintenance Records}

Records of maintenance of equipment shall be suitably recorded. Routine maintenance may be indicated by labels on equipment. Maintenance which results in modification of equipment must be described in sufficient detail and recorded in the operational manual for the particular equipment (see $6.4)$.

\subsection{Instrument Manuals}

A copy of the operational manual supplied with equipment shall be maintained in the or other locations as approved. In the latter case, addition, requires that a copy be placed in the Library. The file will contain descriptions of any modifications that will alter either the operation or servicing instructions furnished by the supplier. As appropriate, the file will contain additional information on operational procedures and/or servicing as may be generated as a result of laboratory experience with the equipment.

Records similar to those described above will be prepared and maintained for equipment built within the laboratory or within

\section{Control Charts}

Control charts are recognized as a prime means for documenting the statistical control of a measurement process.

It is the policy of the that each measurement competence shall develop and maintain appropriate control charts for sub-operations and/or overall operations, where feasible. 
It is the responsibility of the senior scientist in each competence area to Identify the kinds of control charts that will be most useful. When control charts are specified in a given protocol, they shall be maintained in a real-time mode as far as possible and shall be the basis for corrective actions when indicated.

\section{Quality Assessment}

\subsection{Data Verification}

All data reported must include a statement of the uncertainty pertaining to it, and the work plan must include a means for the determination of such limits. In addition, all data will be verified by one or more of the following methods:

1. Simultaneous measurement of an SRM or other certified reference sample

2. Measurement by an independent technique

3. Measurement by an independent operator/apparatus

4. Spiking/recovery technique

5. Replicate measurements by a recognized technique.

The verification data or a summary thereof will become a part of the test report.

The signer of each report, in doing so, verifies that the quality assurance requirements associated with it have been fully met.

8.2 Any significant uncertainties in the measurement process or in the data produced by it will be resolved before data may be reported or released.

\section{Data Review/Reporting}

All data will be subject to peer review before external release. All releases will be in writing. Oral, including telephone, releases are permitted when appropriate, provided they are subsequently (within 24 hours) confirmed in writing, and provided the data have been previously reviewed and approved as herein set forth. The lowest level of review for release of data is by the supervisor or his designate. In the case of sensitive data, a higher level of review may be required. Publication of data is predicated upon editorial review. Data obtained for a sponsor is considered to be the property of the sponsor and its dissemination is subject to the sponsor's release (within the Freedom of Information Act principles).

Reports will be prepared in a format suited to the particular end use. Each report will contain, as far as practical, the following information:

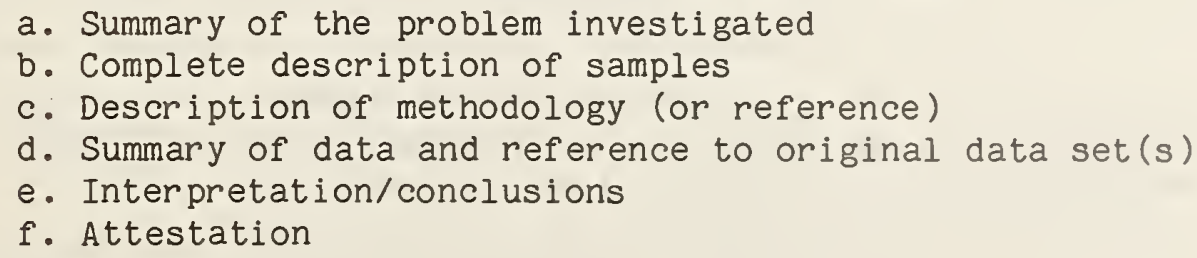

Signatures of analysts(s), Supervisor (or designate(s)). 


\section{Implementation}

Each and every member of the staff has responsibility for the implementation of the quality assurance program, commensurate with her/his technical and/or supervisory and administrative responsibilities. Specific responsibilities are described throughout this document. The general aspects are summarized below.

Individuals: It is the responsibility of each individual to support the quality assurance program in principle and detail. Each member of the staff is responsible for the technical quality of her/his work. All work must be done with the highest level of integrity and professional competence. Records must be kept meticulously. Each member of the staff must be ever alert to problems or sources of error that could compromise the quality of technical work.

Management Chain: The management has the responsibility for supervising and administrating the quality assurance program and is responsible for the overall administration of the program with responsibilities detailed down through the supervisory chain. Specific responsibilities are critical elements of the performance agreements of each supervisory and administrative official.

Quality Assurance Coordinator: The has established the position of Quality Assurance Coordinator for oversight of its quality assurance program. The Coordinator reports to the Director and has the following responsibilities:

On-going review of the individual quality assurance procedures within the various units; provides assistance in the development and implementation of specific quality assurance plans for special analytical programs such as work done for interagency agreements; review of interim and final reports with respect to quality assurance aspects of analytical data.

\section{Research Investigations}

All research investigations shall be carried out efficiently, effectively, and with a high degree of technical excellence. Accurate data are essential for reliable research conclusions. The requirements for acquisition of such data in research situations are similar to those already described. Accordingly, all research investigations shall be carried out consistent with the general principles described above. Quality control during such investigations is primarily the responsiblity of the individual researchers and their supervisors, according to established management chains of supervision and oversight.

Research outputs are in the form of reports and publications. The quality assurance for such outputs is by means of the editorial review process. The respective responsibilities in this process are as follows: 
Investigator/Author: Accuracy of data; adequate support for all conclusions; full disclosure and description of all significant technical details; maintenance of records of all experimental work, including data; safeguarding all data and records for reference in the case of future questions.

Middle Manager: Technical review of all work submitted as reports or publications for scientific/technical adequacy and reliability; certification to higher authority by signing of test reports or publication records that the technical aspects are sound and that supporting data and records are maintained according to prescribed policy.

Upper Manager: Editorial review of all manuscripts, directly or by delegation, to ascertain that their quality meets minimum standards, that all reasonable care has been taken to insure technical validity, and that the contents may be released for external dissemination.

Approved:

Date:

\section{A.2 DEVELOPMENT OF A QA PROTOCOL FOR A SPECIFIC PURPOSE}

Outline of the Development/Implementation Process

Study/Review Analytical Problem

Develop/Review Model

Develop/Review Experimental Plan

Sampling

Measurement/Calibration

Data handling

Develop/Choose/Review Appropriate Technology

Sampling/Calibration/Measurement

Test Proposed Technology for Appropriateness

Validation of methodology

Ident ify Discrete Steps in Technology

In-depth Analysis of Technology/Methodology for Sources of Error

Bias

Variance

Develop/Review Procedures to Reduce Uncertainties to Acceptable Limlts Develop/Specify/Review Quality Control Procedures 
GLPS

GMPS

SOPS

Develop/Specify/Review Quality Assessment Techniques

Reference Materials (RMs)

Control Samples (CSs)

IRMs

Duplicates/Replicates

Spikes

Surrogates

Frequency of Use of CSs, SRMs

Control Charts

Develop/Specify/Review Overall QA plan

Develop/Specify/Review Criteria for

Qualification/Selection of Contract

Laboratories

Monitor Performance

Reference Laboratory Function

Trouble Shoot Follow-up R \& D as Required

A.3 OUTLINE-CONTENT OF A QUALITY ASSURANCE PROTOCOL FOR A SPECIFIC PURPOSE (PSP)

1. Introduction

2.0 Project Description

2.1 Statement of problem

Brief statement of problem with reference to contract, proposal, or other documents where further information can be found.

2.2 Model

Brief discussion of rationale, approach, assumptions

2.3 Major Elements

The basic activities including sampling, measurement, data processing and any other items that will be pursued concurrently.

2.4 Organization

2.4.1 Sponsoring organization

2.4.2 Principal investigator 
2.4.3 Organizational chart and/or other documentation that will describe the respective responsibilities of project/ organizational personnel with respect to present project.

\subsubsection{Priorities/Scheduling}

A statement of any special priorities and/or scheduling pertinent to the present project.

3.0 Work Plan

3.1 Sampling

Kinds and numbers of samples; sampling plan; sampling schedule; sampling methodology; sample storage, preservation, processing; chain of custody. Refer to section 7.1.1 as appropriate.

\subsection{Calibration}

Kinds; frequency; calibrants to be used refer to section 7.1 .2 as appropriate.

\subsection{Measurement}

Kinds of measurements; measurement plan; measurement schedule; measurement methodology; refer to section 7.1 .2 as appropriate.

4.0 Quality Assurance Objectives

4.1 Expected precision and accuracy

4.2 Implementation Summary

A brief summary of the various aspects of the quality assurance plan and how it is to be carried out. Designation of quality assurance responsibilities. Designation of a Quality Assurance Officer as pertinent and responsibilities.

\subsection{Quality Assurance Procedures}

\subsection{Preliminary Exercises}

5.1.1 Statement of any specific training required and how it will be given and evaluated.

5.1.2 Workshop or other indoctrination.

\subsubsection{Performance Checks}

Statement of the measurements/audits that will be required to qualify a laboratory and its personnel and verify their capability to perform or to meet minimum qualification requirements. 
5.2 Quality Assessment Samples

5.2.1 Description

Description of QA samples that will be used with associated documentation.

5.2 .2 Use

5.2.2.1 Mode

5.2.2.2 Frequency

5.3 Control Charts

5.3.1 Specification

Specification of all charts to be maintained and the respective responsibilities for them.

\subsubsection{Strategy}

How control chart information is to be used to provide quality assurance; remedial actions to be taken when necessary.

\subsection{Data Validation}

A statement of the requirements that must be met before data is considered to be valid and may be released for internal or external use, together with who is authorized to make such decisions.

\subsection{Management Practices}

6.1 Chain-of-Custody

Procedures to be followed to safeguard samples; retention procedures; positive identification requirements.

\subsection{Document Control}

Records required; numbering/identification scheme; distribution; document control; storage of records and data.

\subsection{Data Management}

Responsibilities for acquisition; review procedures; release procedures.

\subsection{Reporting}

Scope of reports; schedule for reporting; responsibilities for reporting; responsibilities for release. 
7.1 Methodology

\subsubsection{Sampling}

Specific methodology to be used by reference; specific modifications of existing methods; complete details for unreferenced methods. This shall include: equipment for sampling; method of sampling; pretreatments such as sieving or blending; drying; preparation of analytical samples if not included in 7.1 .2 . (see 3.1 ).

7.1.2 Measurement

Include the same type of information as described in 7.1 .1 .; describe calibrations, calibrants to be used. (see 3.2.; 3.3).

7.2 Maintenance of Facilities and Equipment

\subsubsection{Maintenance Schedules}

7.2.2 Calibration Schedules

7.2.3 Trouble Shooting

This shall include general directions or diagnosis of trouble and corrective actions to be followed.

\section{A.4 OUTLINE - ORGANIZING AND IMPLEMENTATION OF A MONITORING PROGRAM}

Contract/Participant Laboratory Requirements

Pre-Contract/Participation Requirements* In-house QA Program

Developed, in-place, and implemented

Availability of appropriate facilities and equipment**

Availability of trained personnel

Prior experience with methodology**

Prior experience with similar samples***

Pre-initiation-of-work Requirements*

Pre-inspection/review of facilities and plans

* General specification should be included in RFP

* Specific specifications and/or options included in RFP

*** Contractor should submit evidence of such with his proposal. This can be based on laboratory history or on tests done especially for this purpose. Initiator should have samples available for distribution free or at cost for use of prospective contractor/participants. 
Development of specific QA plan

Demonstration of competence on typical samples

Monitoring Contractor/Participant Laboratory Performance*

Adherence to Project QA Plan

Performance on replicates/split samples

Performance on proficiency-testing samples

Maintenance of facilities equipment

Retention of personnel or suitable replacement

Adherence to all aspects of contractual/programmatic requirements

Consistency of results with those of referee laboratory****

Consistency with other labs in monitoring groups

Retention of Status

Retention of qualification status and payment for services (as pertinent) contingent on providing such of required quality

\section{A.5 OUTLINE-FUNCTIONS OF A REFERENCE LABORATORY (RL)}

The following outline is furnished to provide guidance when the need for a reference laboratory (RL) in a monitoring program is considered. The requirements that an RL must satisfy are largely independent of the functions it may serve. The latter will depend on the complexity of the program and extent to which the various functions outlined can be furnished adequately by others in the program.

Purpose of an RL

- To provide confidence in data

- To provide continuing surveillance of measurement program and to advise on corrective actions as required

Functions of an RL

- $\mathrm{R}$ and $\mathrm{D}$ services

-Validate existing methodology

-Identify gaps in methodologies

-Develop or improve methodology

- Reference Materials

-Validate existing RMs

-Develop new RMs

- Referee Laboratory Services

-Analyze percentage of samples

- Corroborate key data

- Trouble shoot problems

****Referee laboratory should have clear and indisputable peer qualifications. It should do sufficient work to be able to clearly defend all judgments, including both acceptability and inacceptability of performance/data. 
- Quality Assurance Services

-Advise/design QA program

-Validate QA program

-Design/Test PSP (Program Plans)

-Conduct proficiency tests

-Qualify participants

-Provide statistical services

- Training

-Workshops

-Specific training

-Methodology

-QA program

-Succession training

Requirements for an RL

- Recognized competence

-Dedication to excellence

- Center of excellence

- Broad interdisciplinary capability and experience

- Recognized impartiality

- On-going QA program to back-up its technical findings

Selection of an RL

- Independent laboratory having required qualifications

- Group member

-Rational role

-Must meet minimum requirements 


\section{APPENDIX B. DATA QUALITY OBJECTIVES}

The setting of realistic data quality requirements is a pre-requisite for planning a measurement program. Data of insufficient quality will have little value for problem solving while data of excess quality may provide little if any additional advantages, and may be counter-productive if extra cost is involved. Accordingly, data quality objectives must be established based on cost-effective considerations of needs of the problem and capability of the measurement process. In addition, knowledge of the expected variability of the samples is needed to estimate the number of samples to be taken and the number of measurements to be made of each sample. While the details of the planning process are beyond the scope of the present paper, the considerations involved are presented for general information in the following.

Qualitative data are commonly considered to require an accuracy of about \pm 30 percent; quantitative data used for hypothesis testing should have a minimum accuracy requirement of about \pm 10 percent; semi-quantitative data will lie between these limits. In questions of distinguishing differences, a numerical factor of 3 will be found to be useful. For example, a standard Reference Material certified to $\pm 1 \%$ is expected to be useful for identifying biases of at least $\pm 3 \%$. Multiple independent measurements will reduce the latter value by a factor of $1 / \sqrt{ } \mathrm{n}$. The same principle may be applied to the identification of small differences in other measurement situations. In general, the minimum detectable difference of two measured values (with $95 \%$ confidence) is $3 \mathrm{U} / \sqrt{ } \mathrm{n}$, where $\mathrm{U}$ represents the uncertainty of the measured results and $\mathrm{n}$ is the number of measurements.

In the previous discussion, the uncertainty, $U$, represents that due to both the measurement process $\left(U_{m}\right)$ and the sample variability $\left(U_{S}\right)$. Ordinarily, $U=\left(U_{S}^{2}+U_{m}^{2}\right)^{1 / 2}$ will apply. A detalled discussion of the propagation of the uncertainties due to sampling and measurement, together with the cost considerations in designing sampling and measurement plans is given by Provost (35A).

The uncertainty of the sample composition, $U_{S}$, is largely a statistical problem, although individual samples may be biased, as well. The latter car result from contamination, sample deterioration, and discriminatory sampling procedures. A well-designed sampling pian should minimize all of the above sources of bias.

The uncertainty of the measurement process contributes both systematic (B) and random $(R)$ uncertainties. The former results from such things as improper calibration, blanks, and non-selectivity of the methodology. The latter includes variability in control of critical parameters. All biases should be corrected to the extent possible so that only uncertainties in the corrections remain.

The total uncertainty, $\mathrm{U}_{\mathrm{T}}$, in either the sample composition or in its measurement may be estimated as follows:

$$
\mathrm{U}_{\mathrm{T}}= \pm\left(\mathrm{U}_{\mathrm{B}}+\mathrm{U}_{\mathrm{R}}\right)
$$




$$
\begin{aligned}
& U_{B}=\left(U_{B_{1}}+U_{B_{2}}+\cdots+U_{B_{n}}\right) \\
& U_{R}=\left(U_{R_{1}}^{2}+U_{R_{2}}^{2}+\cdots+U_{R_{n}}^{2}\right)^{1 / 2}
\end{aligned}
$$

The subscripts indicate the several sources of uncertainty in the bias or random error as they are individually identifiable. All uncertainties are ordinarily estimated at the $95 \%$ confidence level. In other than simple or well-defined measurement problems, the collaboration of a competent

statistician is recommended to assist in the development of realistic data quality objectives and in the design of adequate measurement and sampling plans. 
John K. Taylor

National Bureau of Standards

Absolute Method - A method in which characterization is based entirely on physically (absolute) defined standards.

Accreditiation - A formal process by which a labortory is evaluated, with respect to established criteria, for its competence to perform a specified kind(s) of measurement. Also, the decision based upon such a process. When a certificate is issued, the process is often called certification.

Accuracy - The degree of agreement of a measured value with the true or expected value of the quantity of concern.

Aliquant - A divisor that does not divide a sample into a number of equal parts without leaving a remainder; a sample resulting from such a divisor.

Aliquot - A divisor that divides a sample into a number of equal parts, leaving no remainder; a sample resulting from such a divisor.

Analyte - The specific component measured in a chemical analysis; also called analate.

Assignable cause - A cause believed to be responsible for an identifiable change of precision or accuracy of a measurement process.

Blank - The measured value obtained when a specified component of a sample is not present during the measurement. In such a case, the measured value/signal for the component is believed to be due to artifacts, hence should be deducted from a measured value to give a net value due to the component contained in a sample. The blank measurement must be made so that the correction process is valid.

Blind Sample - A sample submitted for analysis whose composition is known to the submitter but unknown to the analyst. A blind sample thus is one way to test proficiency of a measurement process.

Bias - A systematic error inherent in a method or caused by some artifact or idiosyncrasy of the measurement system. Temperature effects and extraction inefficiencies are examples of this first kind. Blanks, contamination, mechanical losses and calibration errors are examples of the latter kinds. Bias may be both positive and negative and several kinds can exist concurrently, so that net bias is all that can be evaluated, except under special conditions.

*For other definitions, and particularly those related to industrial quality assurance, see: "Quality Systems Terminology", ANSI/ASQC Standard A3-1978, 
Bulk sampling - Sampling of a material that does not consist of discrete, identifiable, constant units, but rather of arbitrary, irregular units.

Calibrant - A substance used to calibrate or to establish the analytical response of a measurement system.

Calibration - Comparison of a measurement standard or instrument with another standard or instrument to report or eliminate by adjustment any variation (deviation) in the accuracy of the item being compared.

Cause-Effect Diagram - A graphical representation of the causes that can produce a specified kind of error in measurement. A popular one is the so-called fish bone diagram, first described by Ishikawa, given this name because of its suggestive shape.

Certification - See accreditation.

Central Line - The long-term expected value of a variable displayed on a control chart.

Certified Reference Material (CRM) - A reference material one or more of whose property values are certified by a technically valid procedure, accompanied by or traceable to a certificate or other documentation which is issued by a certifying body.

Certified Value - The value that appears in a certificate as the best estimate of the value for a property of a reference material.

Chance Cause - A cause for variability of a measurement process that occurs unpredictably, for unknown reasons, and believed to happen by chance, alone.

Check Standard - In physical calibration, an artifact measured periodically, the results of which typically are plotted on a control chart to evaluate the measurement process.

Coefficient of Variation - The standard deviation divided by the value of the parameter measured.

Common Cause - A cause of variability of a measurement process, inherent in and common to the process itself, as contrasted to a special cause (def ined).

Comparative Method - A method which is based on the intercomparison of the sample with a chemical standard.

Confidence Interval - That range of values, calculated from an est1mate of the mean and the standard deviation, which is expected to include the population mean with a stated level of confidence. Confldence intervals in the same context also may be calculated for standard deviations, lines, slopes, points. 
Control Limit - The limits shown on a control chart beyond which it is highly improbable that a point could lie while the system remains in a state of statistical control.

Control Chart - A graphical plot of test results with respect to time or sequence of measurement together with limits within which they are expected to lie when the system is in a state of statistical control.

Control Sample - A material of known composition that is analyzed concurrently with test samples to evaluate a measurement process (see also Check Standard).

Composite Sample - A sample composed of two or more increments selected to represent a population of interest.

Cross Sensitivity - A quantitative measure of the response obtained for an undesired constituent (interferrent) as compared to that for a constituent of interest.

Detection Limit - The smallest concentration/amount of some component of interest that can be measured by a single measurement with a stated level of confidence.

Double Blind - A sample, known by the submitter but submitted to an analyst in such a way that neither its composition nor its identification as a check sample are known to the latter.

Duplicate Measurement - A second measurement made on the same (or identical) sample of material to assist in the evaluation of measurement variance.

Duplicate Sample - A second sample randomly selected from a population of interest (see also split sample) to assist in the evaluation of sample variance.

Education - Disciplining the mind through instruction or study. Education is general and prepares tine mind to react to a variety of situations.

Error - Difference betwen the true or expected value and the measured value of a quantity or parameter.

Figure of Merit - A performance characteristic of a method believed to be useful when deciding its applicability for a specific measurement situation. Typical rigures of merit include: selectivity; sensitivity; detection limit; precision; bias.

Good Laboratory Practice (GLP) - An acceptable way to perform some basic operation or activity in a laboratory, that is known or believed to influence the quality of its outputs. GLP's ordinarily are essentially independent of the measurement techniques used.

Good Measurement Practice (GMP) - An acceptable way to perform some operation associated with a specific measurement technique, and which is known or believed to influence the quality of the measurement. 
Gross Sample (also called bulk sample, lot sample) - One or more increments of material taken from a larger quantity (lot) of material for assay or record purposes.

Homogeneity - The degree to which a property or substance is randomly distributed throughout a material. Homogeneity depends on the size of the subsample under consideration. Thus a mixture of two minerals may be inhomogeneous at the molecular or atomic level, but homogeneous at the particulate level.

Incrememt - An individual portion of material collected by a single operation of a sampling device, from parts of a lot separated in time or space. Increments may be either tested individually or combined (composited) and tested as a unit.

Individuals - Conceivable constituent parts of a population.

Informational Value - Value of a property, not certified but provided because it is believed to be reliable and to provide information important to the certified material.

Intercalibration - The process, procedures, and activities used to ensure that the several laboratories engaged in a monitoring program can produce compatible data. When compatible data outputs are achieved and this situation is maintained, the laboratories can be said to be intercalibrated.

Laboratory Sample - A sample, intended for testing or analysis, prepared from a gross sample or otherwise obtained. The laboratory sample must retain the composition of the gross sample. Often reduction in particle size is necessary in the course of reducing the quantity.

Limiting Mean - The value approached by the average as the number of measurements, made by a stable measurement process, increases indefinitely.

Limit of Linearity (LOL) - The upper limit of concentration or amount of substance for which incremental additions produce constant increments of response.

Limit of Quantitation ( $L O Q$ ) - The lower limit of concentration or amount of substance that must be present before a method is considered to provide quantitative results. By convention $L O Q=10 s_{O}$, where $s_{O}$ is the estimate of the standard deviation at the lowest level of measurement.

Lot - A quantity of bulk material of similar composition whose propertles are under study.

Method - An assemblage of measurement techniques and the order in which they are used.

Outlier - A value which appears to deviate markedly from that for other members of the sample in which it occurs. 
Pareto Analysis - A statistical approach to ranking assignable causes according to the frequency of occurrence.

Performance Audit - A process to evaluate the proficiency of an analyst/ laboratory by evaluation of the results obtalned on known test materials.

Population - A generic term denoting any finite or infinite collection of individual things, objects, or events; in the broadest concept, an aggregate determined by some property that distinguishes things that do and do not belong.

Precision - The degree of mutual agreement characteristic of independent measurements as the result of repeated application of the process under specified conditions. It is concerned with the closeness together of results.

Primary Standard - A substance or artifact, the value of which can be accepted (within specific limits) without question when used to establish the value of the same or related property of another material. Note that the primary standard for one user may have been a secondary standard of another.

Probability - The likelihood of the occurrence of any particular form of an event, estimated as the ratio of the number of ways or times that the event may occur in that form to the total number of ways that it could occur in any form.

Procedure - A set of systematic instructions for using a method of measurement or of sampling or of the steps or operations associated with such.

Protocol - A procedure specified to be used when performing a measurement or related operation, as a conition to obtain results that could be acceptable to the specifier.

Protocol for a Specific Purpose (PSP) - Detailed instructions for the performance of all aspects of a measurement program. This is sometimes called a project QA plan.

Quality - An estimation of acceptability or suitability for a given purpose of an object, item, tangible, or intangible thing.

Quality Assessment - The overall system of activities whose purpose is to provide assurance that the quality control activities are being done effectively. It involves a continuing evaluation of performance of the production system and the quality of the products produced.

Quality Assurance - A system of activities whose purpose is to provide to the producer or user of a product or a service the assurance that it meets defined standards of quality. It consists of two separate but related activities, quality control and quality assessment (defined). 
Quality Circle - A small group of individuals with related interests that meets at regular intervals to consider problems or other matters related to the quality of outputs of a process and to the correction of problems or to the improvement of quality.

Quality Control - The overall system of activities whose purpose is to control the quality of a product or service so that it meets the needs of users. The aim is to provide quality that is satisfactory, adequate, dependable, and economic.

Random Sample - A sample selected from a population, using a randomization process.

Reduction - The process of preparing one or more subsamples from a sample.

Reference Material (RM) - A material or substance one or more properties of which are sufficiently well established to be used for the calibration of an apparatus, the assessment of a measurement method, or for the assignment of values to materials.

Reference Method - A method which has been specified as capable, by virtue of recognized accuracy, of providing primary reference data.

Relative Standard Deviation - The coefficient of variation, expressed as a percentage.

Replicate - A counterpart of another, usually referring to an analytical sample or a measurement. It is the general case for which duplicate is the special case consisting of two samples or measurements.

Routine Method - A method used in recurring analytical problems.

Sample - A portion of a population or lot. It may consist of an individual or groups of individuals. It may refer to objects, materials, or to measurements, conceivable as part of a larger group that could have been considered.

Secondary Standard - A standard whose value is based upon comparison with some primary standard. Note that a secondary standard, once its value is established, can become a primary standard for some other user.

Segment - A specifically demarked portion of a lot, either actual or hypothetical.

Selectivity - The ability of methodology or instrumentation to respond to a desired substance or constituent and not to others. It is sometimes quantified as cross sensitivity, which see.

Sensitivity - Capability of methodology or instrumentation to discriminate between samples having differing concentrations or containing differing amounts of an analate.

Significant Figure - A figure(s) that remains to a number or decimal after the ciphers to the right or left are cancelled. 
Special Cause - A cause of variance or bias that is external (not inherent) to the measurement system.

Split Sample - A replicate portion or sub-sample of a total sample obtained in such a manner that it is not believed to differ significantly from other portions of the same sample.

Standard - A substance or material, the properties of which are believed to be known with sufficient accuracy to permit its use to evaluate the same property of another. In chemical measurements, it of ten describes a solution or substance, commonly prepared by the analyst, to establish a calibration curve or the analytical response function of an instrument.

Standard Addition - A method in which small increments of a substance under measurement are added to a sample under test to establish a response function or, by extrapolation, to detemine the amount of a constituent orginally present in the test sample.

Standard Method - A method (or procedure) of test developed by a standardswriting organization, based on consensus opinion or other criteria, and often evaluated for its reliability by a collaborative testing procedure.

Standard Operations Procedure (SOP) - A procedure adopted for repetitive use when performing a specific measurement or sampling operation. It may be a standard method or one developed by the user.

Standard Reference Material - A reference material distributed and certified by the National Bureau of Standards.

Strata - Segments of a lot that may vary with respect to the property under study.

Subsample - A portion taken from a sample. A laboratory sample may be a subsample of a gross sample; similarly, a test portion may be a subsample of a laboratory sample.

Technique - A physical or chemical principle utilized separately or in combination with other techniques to determine the composition (analysis) of materials.

Test Portion (also called specimen, test specimen, test unit, aliquot) That quantity of a material of proper size for measurement of the property of interest. Test portions may be taken from the gross sample directly, but often preliminary operations, such as mixing or further reduction in particle size, are necessary.

Tolerance Interval - That range of values, calculated from an estimate of the mean and the standard derivation, within which a specified percentage of individual values of population (measurements or sample) are expected to lie with a stated level of confidence 
Traceability - The ability to trace the source of uncertainty of a measurement or a measured value.

Training - Formal or informal instruction designed to provide competence of a specific nature.

Uncertainty - The range of values within which the true value is estimated to lie. It is a best estimate of possible inaccuracy due to both random and systematic error.

Validation - The process by which a sample, measurement method, or a piece of data is deemed to be useful for a specified purpose.

Variance - The value approached by the average of the sum of the squares of deviations of individual measurements from the limiting mean. Mathematically, it may be expressed as

$$
\frac{\sum\left(x_{i}-m\right)^{2}}{n} \rightarrow \sigma^{2} \text { as } n \rightarrow \infty
$$

Ordinarily it cannot be known but only its estimate, $s^{2}$, which is calculated by the expression

$$
s^{2}=\frac{\sum\left(x_{i}-\bar{x}\right)^{2}}{n-1}
$$

Warning Limits - The limits shown on a control chart within which most of the test results are expected to lie (within a $95 \%$ probability) while the system remains in a state of statistical control.

Youden Plot - A graphical presentation of data, recommended first by $W$. J. Youden, in which the result(s) obtained by a laboratory on one sample is plotted with respect to the result(s) it obtained on a similar sample. It helps in deciding whether discrepant results are due to random or systematic error. 
NBS-114A (REV. 2.80)

U.S. DEPT. OF COMM.

BIBLIOGRAPHIC DATA

SHEET (See instructions)

1. PUBLICATION OR
REPORT NO.
NBSIR $85-3105$

2. PorformIng Organ. Report No.1

3. Publlcation Date

February 1985

4. TITLE AND SUBTITLE

Principles of Quality Assurance of Chemical Measurements

5. $\operatorname{AUTHOR}(S)$

Dr. John K. Taylor

6. PERFORMING ORGANIZATION (If joint or other than NBS, see in structions)

NATIONAL BUREAU OF STANDARDS

DEPARTMENT OF COMMERCE

WASHINGTON, D.C. 20234

9. SFONSORING ORGANIZATION NAME AND COMPLETE ADDRESS (Street, City, Stote, ZIP)

Center for Analytical Chemistry

B1dg. 222, Rm. A309

National Bureau of Standards

Gaithersburg, MD 20899

10. SUPPLEMENTARY NOTES

Document describes a computer program; SF-185, FIPS Software Summary, is attached.

11. ABSTRACT (A 200-word or less foctual summory of most significont information. If document includes a significont bibliography or literature survey, mention it here)

The general principles of quality assurance of chemical measurements are discussed. They may be classified as quality control -- what is done to control the quality of the measurement process, and quality assessment -- what is done to evaluate the quality of the data output. Quality assurance practices are considered as a hierarchy with levels progressing from the analyst, the laboratory, the project, to the program. The activities of each level are different and depend upon the ones beneath it. 'Recommendations are presented for developing credible quality assurance practices at each level. An appendix contains outlines that may be used to develop the various documents associated with a quality assurance program.

12. KEY WORDS (Six to twelve entries; olphobetical order; copitolize only proper nomes; and separote key words by semicolons)

chemical analysis; calibration; control charts; good laboratory practices; good measurement practices; quality assessment; quality assurance; quality contro 1; standard operations procedures: statistical measurement control

13. AVAILABILITY

X. Unlimited

For Officlal Distribution. Do Not Release to NTIS

Order From Superintendent of Documents, U.S. Government Printing Office, Washington, D.C. 20402.

14. NO. OF

PRINTED PAGES

80

15. Price

\footnotetext{
$\square$ Order From National Technical Information Service (NTIS), Springfield, VA. 2216I
} 

